\title{
Mammary adipocytes stimulate breast cancer invasion through metabolic remodeling of tumor cells
}

Yuan Yuan Wang, ${ }^{1,2,3}$ Camille Attané,, ${ }^{2}$ Delphine Milhas, ${ }^{1}$ Béatrice Dirat,, ${ }^{1,2}$ Stéphanie Dauvillier, ${ }^{1}$ Adrien Guerard, ${ }^{1}$ Julia Gilhodes, ${ }^{4}$ Ikrame Lazar, ${ }^{1}$ Nathalie Alet, ${ }^{5}$ Victor Laurent, ${ }^{1}$ Sophie Le Gonidec, ${ }^{2}$ Denis Biard, ${ }^{6}$ Caroline Hervé, ${ }^{5}$ Frédéric Bost, ${ }^{7}$ Guo Sheng Ren, ${ }^{3}$ Françoise Bono, ${ }^{5}$ Ghislaine Escourrou, ${ }^{8}$ Marc Prentki, ${ }^{9}$ Laurence Nieto, ${ }^{1}$ Philippe Valet, ${ }^{2}$ and Catherine Muller ${ }^{1}$

IInstitut de Pharmacologie et de Biologie Structurale (IPBS), Université de Toulouse, CNRS, ${ }^{2}$ Institut des Maladies Métaboliques et Cardiovasculaires (I2MC), Université de Toulouse, INSERM, UPS, Toulouse, France. ${ }^{3}$ Department of Endocrine and Breast Surgery, The First Affiliated Hospital of Chongqing Medical University, Chongqing, China. ${ }^{4}$ Departement de Biostatistiques, Institut Universitaire du Cancer, Toulouse, France. ${ }^{5}$ EVOTEC France, Toulouse, France. ${ }^{6} \mathrm{CEA}, \mathrm{DSV}$, IMETI/SEPIA, France. 'Institut National de la Santé et de la Recherche Médicale, U1065, Centre Méditerranéen de Médecine Moléculaire, Nice, France. ${ }^{8}$ Service d'Anatomo-Pathologie, Institut Universitaire du Cancer, Toulouse, France. ${ }^{9}$ Departments of Nutrition and Biochemistry and Montreal Diabetes Research Center, CRCHUM and Université de Montréal, Montréal, Quebec, Canada.

In breast cancer, a key feature of peritumoral adipocytes is their loss of lipid content observed both in vitro and in human tumors. The free fatty acids (FFAs), released by adipocytes after lipolysis induced by tumor secretions, are transferred and stored in tumor cells as triglycerides in lipid droplets. In tumor cell lines, we demonstrate that FFAs can be released over time from lipid droplets through an adipose triglyceride lipase-dependent (ATCL-dependent) lipolytic pathway. In vivo, ATCL is expressed in human tumors where its expression correlates with tumor aggressiveness and is upregulated by contact with adipocytes. The released FFAs are then used for fatty acid $\beta$-oxidation (FAO), an active process in cancer but not normal breast epithelial cells, and regulated by coculture with adipocytes. However, in cocultivated cells, FAO is uncoupled from ATP production, leading to AMPK/acetyl-CoA carboxylase activation, a circle that maintains this state of metabolic remodeling. The increased invasive capacities of tumor cells induced by coculture are completely abrogated by inhibition of the coupled ATCL-dependent lipolysis/FAO pathways. These results show a complex metabolic symbiosis between tumor-surrounding adipocytes and cancer cells that stimulate their invasiveness, highlighting ATCL as a potential therapeutic target to impede breast cancer progression.

Authorship note: CA and DM contributed equally to this work. LN and PV contributed equally to this work.

Conflict of interest: The authors have declared that no conflict of interest exists.

Submitted: March 24, 2016 Accepted: January 17, 2017 Published: February 23, 2017

Reference information: JCI Insight. 2017;2(4):e87489. https:// doi.org/10.1172/jci.insight.87489.

\section{Introduction}

Cancer tissues exhibit greater metabolic plasticity than normal tissues because they must survive in a dynamic environment where oxygen and nutrients are often scarce $(1,2)$. In human tumors, both mitochondrial oxidative phosphorylation (OXPHOS) and aerobic glycolysis coexist, their relative shares being dependent on both the genetic background of the tumors and their microenvironment (3-5). In addition to glucose and glutamine, free fatty acids (FFAs) are an important energy source, through mitochondrial fatty acid oxidation (FAO) (6). At physiological levels, FAO is carried out in energy-consuming tissues (such as the heart and skeletal muscle). Recent works have highlighted a role for this metabolic pathway in cancer cells to ensure a rapid energy supply in response to environmental changes (6). In particular, FAO is induced during loss of attachment (LOA) of epithelial tumor cells, rescuing them from death by anoikis (7). Recently, Carracedo and colleagues have described a novel mechanism in which FAO is regulated by the promyelocytic leukemia (PML) protein and, again, the ability of PML to increase FAO activity promoted cell survival on LOA in cancerous epithelial cells (8). In lung cancers, the expression of an atypical isoform of carnitine palmitoyltransferase 1, CPT1C, promotes FAO, ATP production, and tumor growth, and it rescues cells from metabolic stress (8). Increased tumor aggressiveness due to FAO is not necessarily 
linked to ATP production in all cancer cells. For instance, in a subset of leukemias, FAO is required for cell survival independently of ATP production and may influence BAX- and BAK-dependent mitochondrial permeability transition pore formation (9). FAO might also lead to respiratory chain uncoupling, defined as the inability of cells to synthesize ATP in response to the mitochondrial proton gradient (9).

Cancer cell metabolism is also regulated by direct crosstalk with tumor-surrounding stromal components. One important source of FFAs to fuel tumor cell FAO could be tumor-surrounding adipocytes. Indeed, we have previously demonstrated in breast cancer that, in vitro and in vivo, adipocytes exhibit a loss of lipid content, a decreased expression of adipocyte markers, and an activated state indicated predominantly by the overexpression of proinflammatory cytokines (10). We named these cells cancer-associated adipocytes (CAA) (10-12). We have further demonstrated that, upon prolonged coculture with tumor cells, almost all lipid droplets disappear from adipocytes, resulting in morphological changes toward a fibroblast-like shape (13). The loss of lipid content in tumor-surrounding adipocytes suggests that FFAs could be released from these cells and transferred to cancer cells. Such a direct transfer of lipids has been demonstrated in both prostate (14) and ovarian cancer (15). In their elegant study, Nieman et al. demonstrated that lipid transfer from adipocytes to cancer cells fuels tumor growth in vitro and in vivo (15). However, the mechanism linking these adipocyte-derived FFAs and increased tumor progression is not fully elucidated. The fact that ovarian cancer cells exhibit FAO activity, which is slightly upregulated in the presence of adipocytes, suggests - without demonstrating it directly - that these FFAs are used as an energy source for cancer cells. Indeed, the effect of FAO inhibition on tumor progression, as well as on ATP production, was not investigated in this model. In the present study, we demonstrate that lipids from tumor surrounding adipocytes promote breast cancer invasion through synergistic adipose triglyceride (TG) lipase-dependent (ATGL-dependent) lipolysis and uncoupled FAO, unraveling the complex metabolic symbiosis between the two cell populations.

\section{Results}

Lipid transfer between adipocytes and tumor cells is associated with increased FAO activity. Firstly, we investigated whether coculture with adipocytes could lead to an increase in lipid accumulation in epithelial breast cancer cells, as previously shown for prostate and ovarian cancer $(14,15)$. Human breast cancer cell lines with low (ZR-75-1) or high (SUM159PT) invasive capacities were used, as we have previously characterized their ability to modify the adipocyte phenotype $(10,13)$. As shown in Figure 1A, cocultivated ZR-75-1 exhibit multiple, small lipid droplets as revealed by 2 probes that stain neutral lipids, either the lipophilic fluorescent dye Bodipy 493/503 (left panel) or red oil (middle panel). Such lipid accumulation in cocultivated cells was associated with less compact colonies and a more scattered aspect when compared with noncocultivated cells (Figure 1A). TG dosage revealed a 5-fold increase in cocultivated cells, in accordance with the lipid droplet accumulation (Figure 1A, right panel). Similar results were obtained with SUM159PT cells (Figure 1B). Total lipids were extracted and analyzed by high-performance thin layer chromatography (HPTLC) and electrospray ionization mass spectroscopy (ESI-MS). In contrast to TG, no changes were observed in diglyceride, cholesterol, and cholesteryl-ester levels, and monoglycerides were undetectable (Supplemental Figure 1A; supplemental material available online with this article; https:// doi.org/10.1172/jci.insight.87489DS1). Saturated fatty acid (SFA) levels were increased in cocultivated cells, whereas unsaturated FA levels were not modified by coculture (Supplemental Figure 1, B-D). Among SFA, a significant increase of both C16:0 and C18:0 was observed (Supplemental Figure 1E). Secondly, several experiments were performed to demonstrate that the lipids accumulating in tumor cells originated from adipocytes. We first demonstrated the presence of a lipolytic process in tumor-surrounding adipocytes. Glycerol, one of the products of TG hydrolysis along with FFAs, was released by adipocytes incubated with tumor cell conditioned medium (CM) prepared both from ZR-75-1 and SUM159PT cells (Figure 1C). It is noteworthy that the CM of normal human mammary epithelial cells (HMEC) was unable to induce this effect, emphasizing that this process is tumor specific (Figure 1C). As shown in Supplemental Figure 2, A and B, mature adipocytes incubated in the presence of tumor cell CM exhibit a reduction in the number and size of lipid droplets, which correlates with a significant decrease in TG content. Furthermore, this lipolytic process occurring in adipocytes in the presence of tumor CM was associated with the activation of Hormone-Sensitive Lipase (HSL), as revealed by its phosphorylation (Supplemental Figure 2C). Catecholamines are considered as major regulators of lipolysis. $\beta$-Adrenergic receptor ( $\beta$-AR) activation elicits a G protein-coupled cascade, leading ultimately to the phosphorylation and activation of HSL (16). 
A

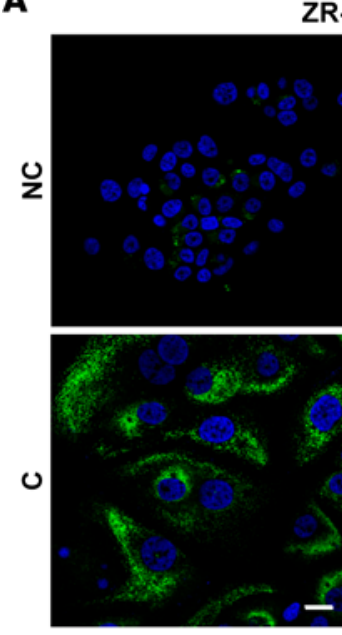

ZR-75-1
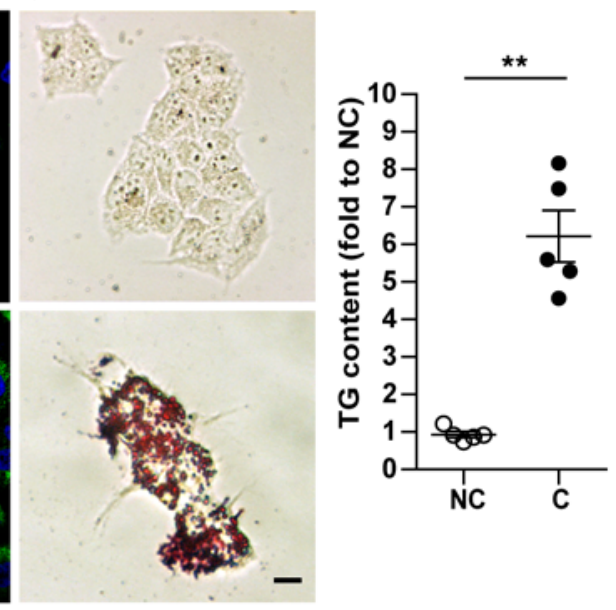

C

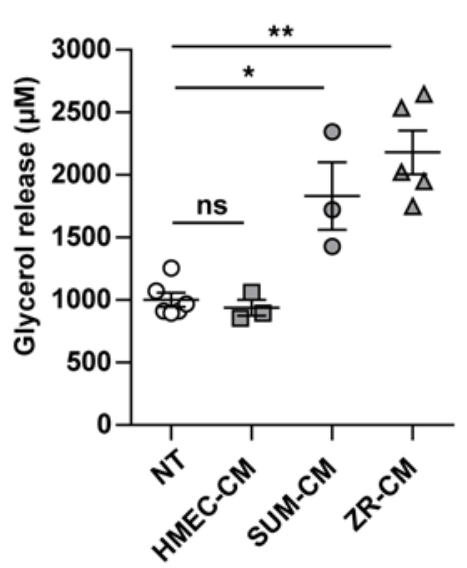

E

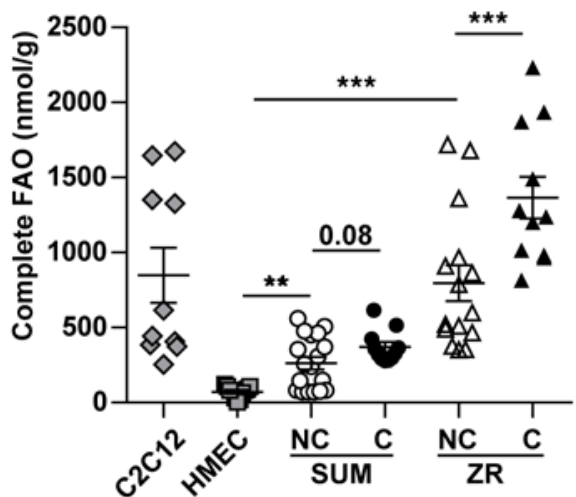

B
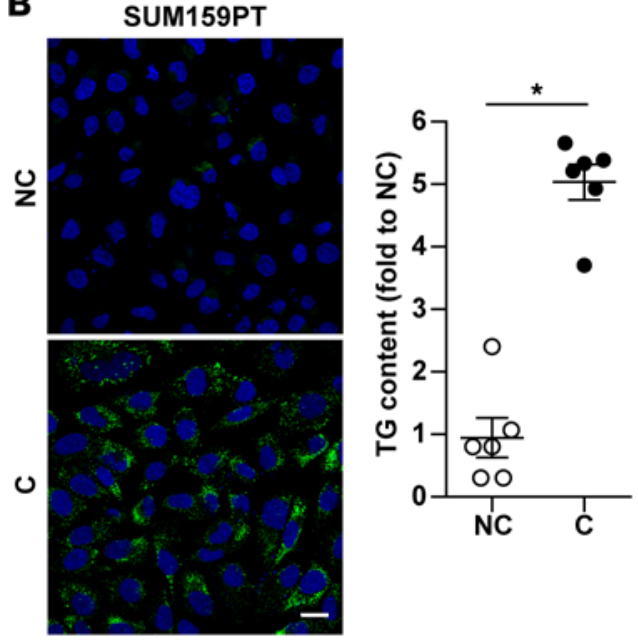

ZR-75-1
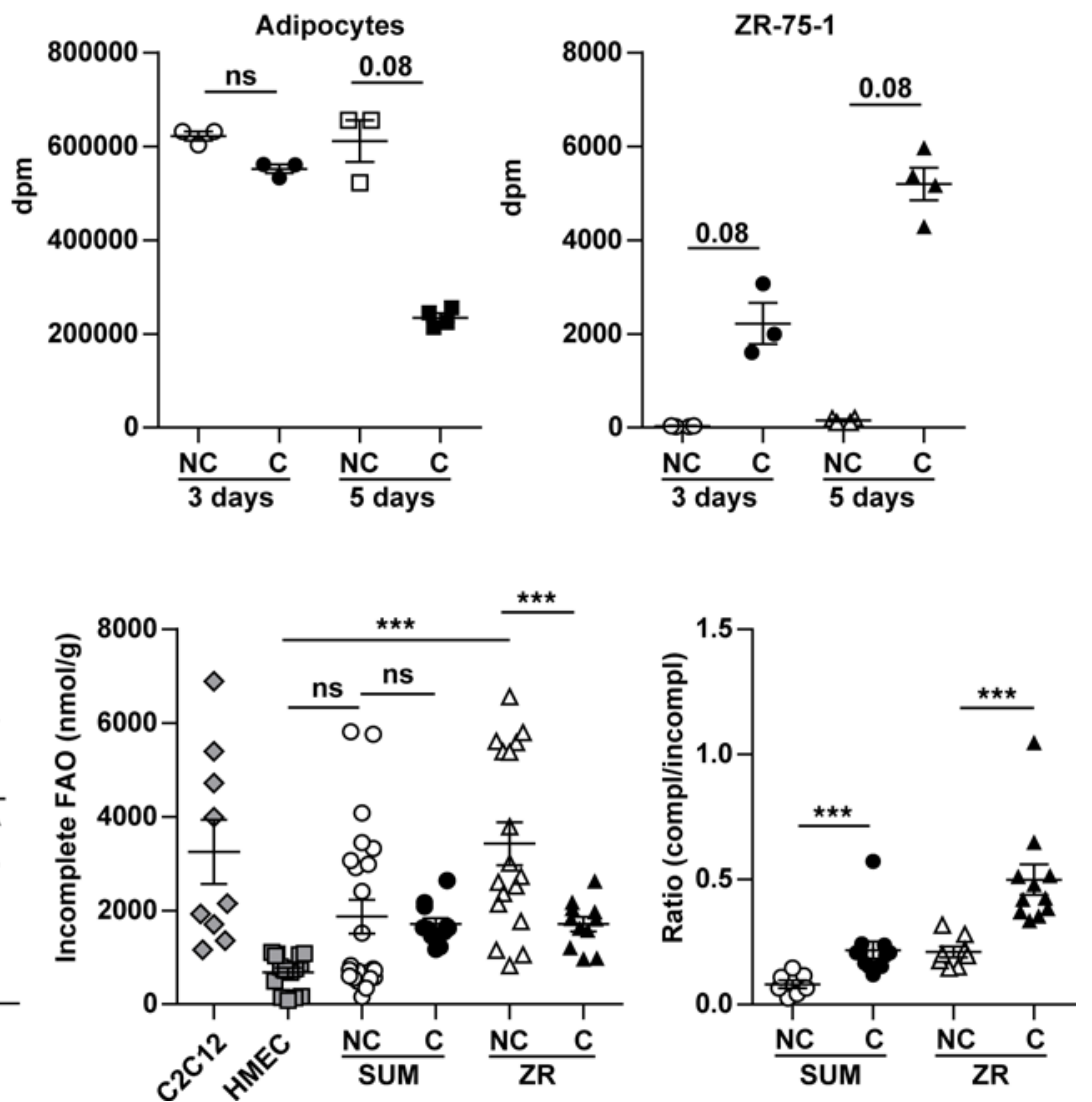

Figure 1. A lipid transfer occurs between adipocytes and cancer cells, which in turn exhibit increased fatty acid oxidation (FAO). (A) Left, lipid accumulation in ZR-75-1 cells cultured alone (NC) or with 3T3-F442A mature adipocytes (C) for 3 days (cells were stained with Bodipy [lipids in green and nuclear counterstaining in blue], left panel or red oil, right panel). Right, triglyceride (TC) content dosed in ZR-75-1 cells cocultivated or not with adipocytes ( $n=$ 5). (B) Lipid accumulation in SUM159PT cocultivated or not with adipocytes, shown after staining with Bodipy (left) or by measure of TC content (right) ( $n$ = 6). (A and B) At least 3 experiments were conducted, and representative experiments are shown. Scale bars: $20 \mu \mathrm{m}$. (C) Mature adipocytes were treated or not (NT) with conditioned medium (CM) from either human mammary epithelial cells (HMEC) or breast tumor cells (ZR, ZR-75-1; SUM, SUM159PT) for 3 days. Cell supernatants were collected, and free glycerol release was measured $(n=3-6)$. (D) Radiolabeled mature adipocytes obtained from in vitro differentiation of 3T3-F442A preadipocytes in the presence of $\left[{ }^{14} \mathrm{C}\right]$ palmitate were cocultivated or not with ZR-75-1 tumor cells for 3 and 5 days. The radioactive content (disintegrations per minute [dpm]) was measured in adipocytes or tumor cells ( $n=3-4)$. (E) Complete (left panel, $n=10-18$ ) and incomplete (middle panel, $n=9-23$ ) FAO in HMEC and indicated tumor cells cultured alone or with adipocytes (the myoblast cell line C2C12 was used as a positive control). The ratio between complete to incomplete FAO is shown (right panel, $n=7-12$ ). Bars and error flags represent means \pm SEM; statistically significant by Student's $t$ test (A and $\mathbf{B}$ ) or Mann-Whitney $U$ test (C-E), ${ }^{*} P<0.05,{ }^{* *} P<0.01,{ }^{* *} P<0.001$. 
A

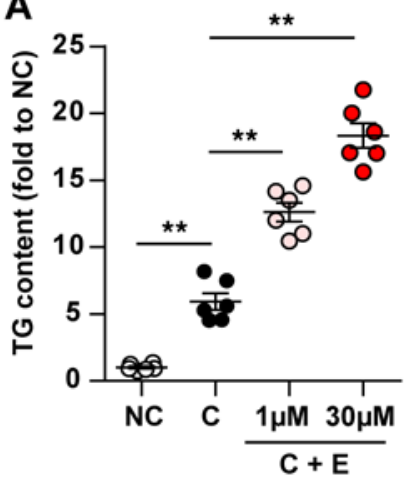

B

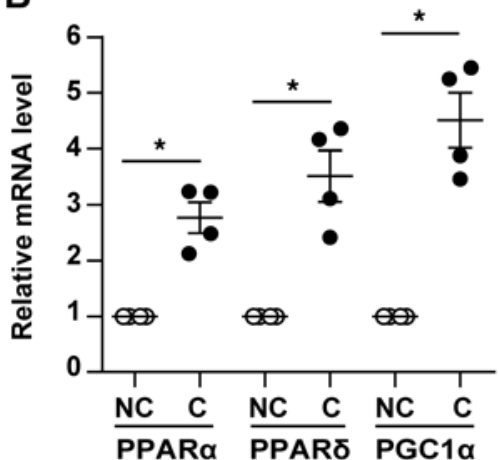

E

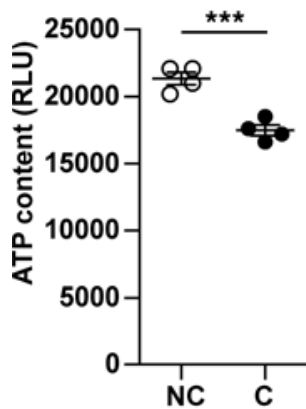

H

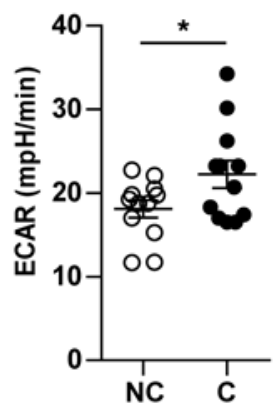

NC
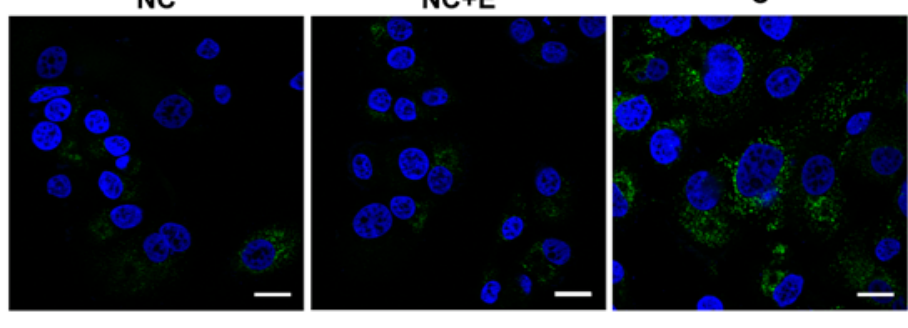

C

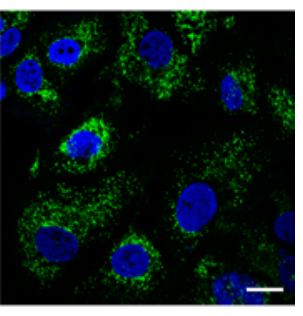

D

C

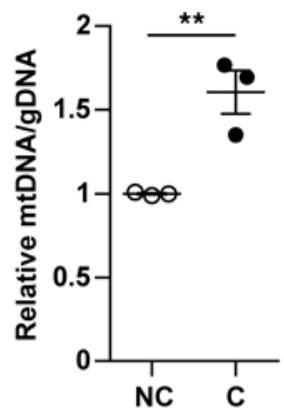

NC
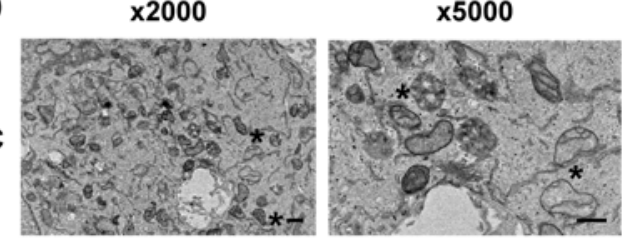

C
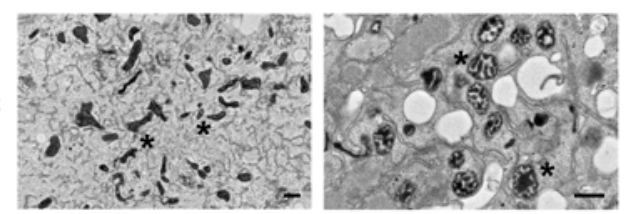

Figure 2. In the presence of adipocytes, breast cancer cells undergo a metabolic switch toward uncoupled mitochondrial fatty acid oxidation (FAO). ZR-75-1 cells were cocultivated (C) or not (NC) with adipocytes for 3 days. (A) Lipid accumulation in tumor cells in the presence $(+E)$ or not of Etomoxir $(1 \mu \mathrm{M}$ and $30 \mu \mathrm{M})$. Left, triglycerides (TG) content $(n=6)$. Right, lipid staining with Bodipy in green and DAPI in blue. Etomoxir $(30 \mu \mathrm{M})$. Scale bars: $30 \mu \mathrm{m}$. (B) Expression of the indicated genes measured by qPCR in tumor cells $(n=4)$. (C) Mitochondrial DNA (ratio of mitochondrial [mtDNA] to genomic [gDNA] DNA levels) levels in tumor cells $(n=3)$. (D) Representative experiments of transmission electron microscopy highlighting mitochondrial ultra-structural changes $\left(^{*}\right)$ in C or NC. Scale bars: $1 \mu \mathrm{m}$ and $2 \mu \mathrm{m}$. (A and D) At least 3 experiments were conducted, and representative images are shown. (E) Total ATP production in tumor cells $(n=4)$. (F) ATP generated by glycolysis in tumor cells $(n=4)$. (C) Lactate released by tumor cells $(n=6)$. (H) Extracellular acidification rate (ECAR) evaluated after addition of $10 \mathrm{mM}$ glucose to tumor cells $(n=12)$. (I) Oxygen consumption rate (OCR) was measured in the presence of palmitate and coupled respiration, proton leak and nonmitochondrial respiration were calculated as described in Methods $(n=9-12)$. Bars and error flags represent means \pm SEM; statistically significant by Mann-Whitney $U$ test (A, B and I) or Student's $t$ test $(\mathbf{C}-\mathbf{H}),{ }^{*} P<0.05,{ }^{* *} P<0.01,{ }^{* * *} P<0.001$.

Contrarily, by binding to $\alpha 2-\mathrm{AR}$, catecholamines could also exert a major anti-lipolytic effect, which is abrogated in the presence of $\alpha 2$-AR antagonists (16). As shown in Supplemental Figure $3 \mathrm{~A}$, we found that neither propranolol (a $\beta$-AR antagonist) nor UK14304 (an $\alpha 2$-AR agonist) were able to inhibit the lipolysis induced by tumor secretions, therefore eliminating the role of catecholamines in this process. The roles of 2 other well-known lipolytic factors, IL6 and TNF $\alpha$ (17), were investigated. Only IL6 was detected in the supernatant of the 2 cancer cell lines used (18). However, the lipolysis induced by tumor secretions 
A

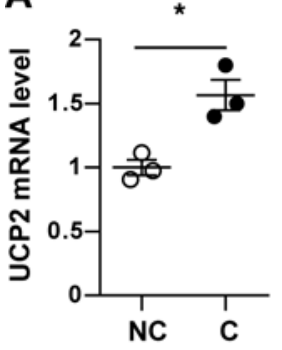

B

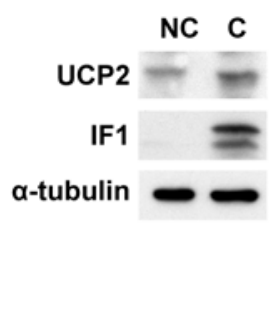

C

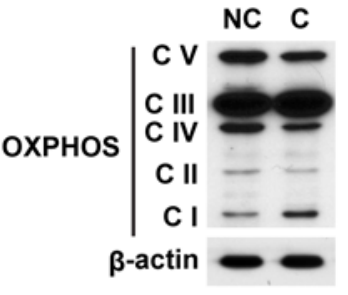

D

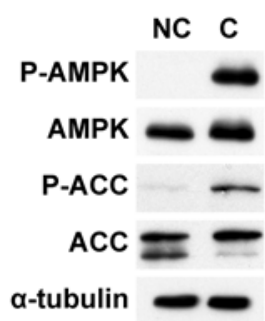

E
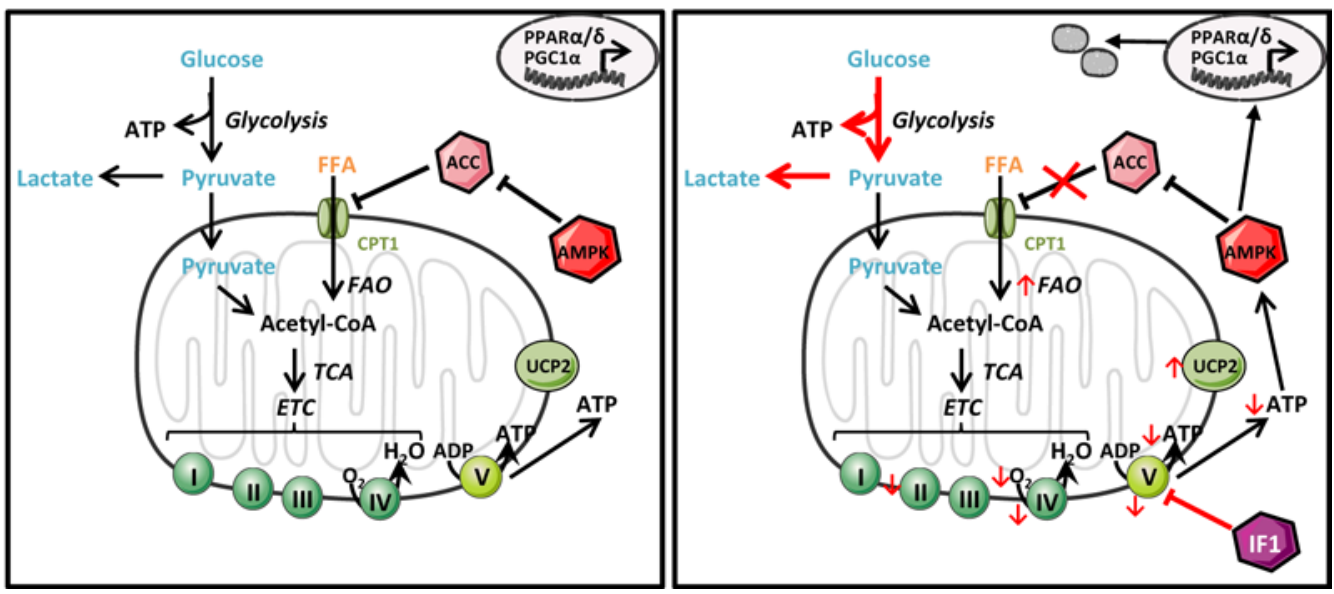

Figure 3. Peritumoral adipocytes induce a metabolic remodeling in favor of uncoupled fatty acid oxidation (FA0). ZR-75-1 cells were cocultivated (C) or not (NC) with adipocytes for 3 days. (A) Quantification of UCP2 mRNA levels $(n=3)$. (B) UCP2 and inhibitory factor 1 (IF1) expression evaluated by Western blot. $\alpha$-Tubulin is used as a loading control. (C) Expression of mitochondrial oxidative phosphorylation (OXPHOS) complexes evaluated by Western blot. Actin is shown as a control for equal protein loading. (D) Expression of total and phosphorylated forms of the acetyl-CoA carboxylase (ACC) and AMPK. $\alpha$-Tubulin is used as a loading control. (B-D) At least 3 experiments were conducted, and representative experiments are shown. (E) Schematic representation of the metabolic remodeling, which takes place in tumor cells in the presence of adipocytes. Bars and error flags represent means \pm SEM; statistically significant by Student's $t$ test, ${ }^{*} P<0.05$.

was unaffected by an anti-IL6 blocking monoclonal antibody (Supplemental Figure 3B). To determine the source of lipids detected in cancer cells after coculture and to rule out the possibility of de novo lipogenesis in cancer cells, we cultured cancer cells for 3 or 5 days with adipocytes that had been differentiated in the presence of radiolabeled palmitic acid. We observed a progressive decrease of radioactivity in adipocytes correlated with a progressive increase of radioactivity contained in tumor cells (Figure 1D). Taken together, these results show that a bidirectional crosstalk takes place between breast tumors and surrounding adipocytes, that favors a transfer of lipids between the 2 populations.

We then questioned whether coculture with adipocytes promotes FAO in cancer cells. As shown in Figure 1E, we found that the 2 tumor cell lines used were able to perform FAO at significantly higher levels than HMEC cells, underlining the potential importance of this metabolic pathway for tumor cells. Coculture with adipocytes increases the ability of tumor cells to completely oxidize FA, whereas incomplete FAO decreases (Figure 1E, left and middle panel). As a result, the oxidative efficiency was significantly elevated in cocultivated cells as compared with noncocultivated cells (Figure 1E, right panel). Our results show a lipid transfer between adipocytes and tumor cells leading to storage of excess FFAs as TG in breast tumor cells, which is accompanied by an upregulation of FAO. However, these results are only correlative and do not directly assess the fate of these lipids in tumor cells.

Increased FAO in cocultivated cells is not coupled to ATP production. To begin, we investigated whether the adipocyte-derived FFAs were used for FAO, using the pharmacological FAO inhibitor etomoxir (ETO). ETO inhibits the entry of FFAs into mitochondria by blocking the activity of the long-chain 
FA transporter, carnitine palmitoyl transferase 1 (CPT1) (19). As a preliminary step, we verified that, in ZR-75-1 cells, complete FAO was efficiently inhibited by ETO in a dose-dependent manner (Supplemental Figure 4). When tumor cells were cocultivated with adipocytes in the presence of ETO, a 2- to 3-fold increase in TG content was observed compared with untreated cells (Figure 2A, left panel). This increase in lipid content is also illustrated by the staining performed with bodipy (Figure 2A, right panel). Similar results were obtained with SUM159PT cells (Supplemental Figure 5). We then investigated the cellular processes known to positively regulate FAO activity. Interestingly, a significant increase in the levels of the transcriptional coactivator PGC1A (PPAR $\gamma$ coactivator $1 \alpha$ ) was observed in ZR-75-1 cells cocultivated for 3 days with adipocytes compared with tumor cells grown alone. Similar overexpression was observed for PGC1 $\alpha$-associated transcription factors PPARA and -D (Figure 2B). In SUM159PT, a significant upregulation of PPARA was observed in cocultivated cells without regulation of the level of PGC1A (Supplemental Figure 6, A-C), a result that could be explained by the high basal expression of this transcription factor in noncocultivated cells (Supplemental Figure 6D). An increased ratio between mitochondrial and genomic DNA was observed in cocultivated breast cancer cells as compared with noncocultivated cells (1.6- to 2-fold) (Figure 2C and Supplemental Figure 6E). Substantial changes in the ultrastructure of mitochondria (i.e., increased matrix density and expansion of the cristal spaces), known to correlate with an increase in their activity (20), were also found in cocultivated ZR-75-1 tumor cells (Figure 2D). Altogether, these results show that adipocytes stimulate complete FAO in breast cancer cells by increasing mitochondria biogenesis and function. We then tested whether increased FAO in cocultivated cells is associated with increased ATP production. Surprisingly, we observed a significant decrease of global ATP in cells cultivated in the presence of adipocytes (Figure 2E). Both glycolytic ATP and lactate production were increased under coculture conditions (Figure 2, F and G), suggesting that coculture induced a shift toward anaerobic glycolysis. Accordingly, the extracellular acidification rate (ECAR) in response to glucose $(10 \mathrm{mM})$ was increased after coculture (Figure $2 \mathrm{H})$. Addition of oligomycin (an inhibitor of the ATP synthase in the electron transport chain [ETC]) stimulates ECAR in control cells but not in cocultivated cells, demonstrating that anaerobic glycolysis is already maximal in cocultivated cells in the presence of glucose (Supplemental Figure 7A). In parallel, we observed a decrease in the oxygen consumption rate (OCR) at basal level and in the presence of glucose in cocultivated cells, and oligomycin only had a very slight effect, demonstrating that the ETC is less active and not coupled to ATP production in these conditions (see Supplemental Figure 7B). We then investigated OCR in conditions that favor FAO (Krebs medium with palmitate, carnitine, and restricted glucose). In these conditions, OCR was decreased in cocultivated cells as compared with noncocultivated cells, and oligomycin had almost no effect on OCR in cocultivated cells, highlighting again that, in these cells, the ETC is less active and not coupled to ATP production (Figure 2I and Supplemental Figure 7C). The proportion of basal respiration associated with ATP production, a proton leak or nonmitochondrial respiration was calculated as previously described (21). The results indicated that, in cocultivated cells, the proton leak and nonmitochondrial respiration (performed by NADPH oxidases) are increased at the expense of ATP production (Figure 2I). All these observations indicate that, in cocultivated cells, increased FAO is not coupled to ATP production and suggest that uncoupling and nonmitochondrial respiration are increased.

To evaluate whether increased expression of uncoupling proteins contributed to the observed metabolic remodeling, we examined the expression of the uncoupling protein 2 (UCP2) and mitochondrial membrane potential $(\triangle \Psi)$. UCP2 mRNA and protein expression were increased in cocultivated cells (Figure 3, A and B), whereas a significant decrease of $\Delta \Psi$ was observed after coculture (Supplemental Figure 8), underlining the implication of uncoupled respiration in cocultivated cells. As soon as mitochondria become uncoupled, $\Delta \Psi$ falls and F0F1-ATP synthase can reverse its function to maintain $\Delta \Psi$ at the expense of ATP consumption. Cells can attenuate this fall in $\Delta \Psi$ by decreasing the expression of F0F1-ATP synthase subunits and by increasing the expression of its endogenous inhibitor (inhibitory factor 1, IF1), which specifically inhibits F0F1-ATP synthase $(22,23)$. In agreement with the hypothesis of mitochondrial uncoupling, coculture with adipocytes also induced an increase in IF1 expression as compared with control cells (Figure 3B). We then completed profiling of the metabolic signature in cocultivated compared with noncocultivated cells by evaluating the quantity of ETC complexes I to IV and F0F1-ATP synthase (complex V) by Western blot (Figure 3C). Interestingly, we observed an increase of complex I with a downregulation of mitochondrial OXPHOS complexes II, IV, and V. The decreased expression of F0F1-ATPase subunits (as well as the lack of inhibition of OCR by oligomycin, Supplemental Figure 7) and the concomitant increase in IF1 
expression consolidate the hypothesis that this mitochondrial uncoupling in cocultivated cells occurs in concomitance with F0F1-ATP synthase inhibition. In response to the fall in cellular ATP content (increase of AMP/ATP ratio) in cocultivated cells, AMPK is activated, as evidenced by increased phosphorylation on the conserved Thr172 residue in the activation loop of catalytic subunits (Figure 3D). A crucial process activated by AMPK is mitochondrial biogenesis, which, in the long-term, generates increased capacity for oxidative catabolism of both glucose and FAs (22). Another step promoted by AMPK is the uptake of FAs into mitochondria, which is the rate-limiting step in $\beta$-oxidation, via phosphorylation and inactivation of the acetyl-CoA carboxylase (ACC) (22). As shown in Figure 3D, an increase in ACC phosphorylation, indicating its inactivation, was observed in cocultivated cells. Therefore, in cocultivated cells, the uncoupling of FAO and the concomitant shift toward anaerobic glycolysis leads to a decrease in ATP content, which in turns increases AMPK activity to induce mitochondrial biogenesis and promote, through ACC phosphorylation, uncoupled FAO, leading to a persistent state of metabolic remodeling (see Figure 3E).

Inhibition of FAO by pharmacological agents and genetic strategies specifically hampers invasiveness of cocultivated breast cancer cells in vitro and in vivo. We have previously demonstrated that coculture with adipocytes stimulates cell invasion (10). In light of this, we investigated the role of uncoupled FAO on tumor aggressiveness using ETO. As shown in Figure 4A (left panel), the invasion of ZR-75-1 tumor cells toward medium containing $10 \%$ FCS was increased by 3 -fold in cells previously cocultivated with adipocytes as compared with tumor cells grown alone, and this effect was completely abrogated by ETO. Noteworthy, ETO exposure had no effect on the invasion of noncocultivated cells (Figure 4A). Similarly, ETO inhibited SUM159PT invasion after coculture, although adipocyte-induced invasion was less pronounced in these aggressive cells, as previously described (10) (Figure 4A, right panel). By contrast, ETO was without effect on cell proliferation as determined by cell number and cell cycle distribution in cocultivated-treated cells as compared with control-treated cells (Supplemental Figure 9, A and B). We ruled out the possibility that increased tumor cell invasion was due to anaerobic glycolysis and lactacte production - not to uncoupled FAO - by using a specific inhibitor of glycolysis. We indeed used 2-deoxyglucose (2DG), which inhibits hexokinase, the first enzyme required for this pathway (24). Treatment of cocultivated cells with 2DG decreased lactate production but increased cellular invasion (Supplemental Figure 10, A and B). Therefore, during coculture, adipocytes stimulate the invasiveness of tumor cells, and this effect is specifically abrogated by inhibition of FAO by ETO. To test the hypothesis that priming tumor cells with adipocytes regulates distal organ seeding and growth of tumor cells in vivo, and that this effect could be interrupted by FAO inhibition, we performed tail vein metastasis assays using the TS/A cell line. It has been previously shown that, when injected i.v. in syngenic immunocompetent BALB/c mice, these cells form metastases in lungs and liver after 1-2 weeks (25). Before performing these in vivo experiments, we assessed that TS/A cells were a suitable model. As shown in Supplemental Figure 11, these cells exhibited an increase in lipid content when cocultivated with adipocytes, an increase in the ratio of complete to incomplete FAO, as well as a decrease in invasiveness in the presence of ETO in vitro. Untreated or ETO-treated TS/A tumor cells were cultured in vitro in the presence or not of adipocytes, then harvested and injected i.v. into BALB/c host mice. As shown in Figure 4B, the number of lung metastases was enhanced in mice injected with TS/A cells previously cocultivated with adipocytes as compared with mice injected with TS/A grown alone, and this effect was abrogated in the presence of ETO. We have previously demonstrated that ZR-75-1 cells cocultivated with adipocytes exhibit incomplete epithelial to mesenchymal transition (EMT) (10). As shown in Figure 4C, all the hallmarks of EMT observed in cocultivated cells — such as a more scattered aspect and less compacted colonies, the development of multiple extended protrusions shown by $\beta$-actin staining, and the downregulation of membrane E-cadherin expression to a disorganized state in the cytoplasm - were inhibited by ETO treatment. Several transcription factors, known as EMT inducers, act as transcriptional repressors of E-cadherin and directly modulate the expression of many genes involved in cancer invasion and metastasis, consequently promoting EMT in vitro (26). These transcription factors include members of the snail family, and it has been proposed that SNAI1 (also named SNAIL) plays a role in inducing the first EMT steps that lead to the initiation of the invasive process (27). As shown in Figure 4D, SNAI1 expression was increased in both ZR-75-1 and M159PT cocultivated cells, and this overexpression was inhibited in ETO-treated cells.

As shown before, FAO is controlled by FA import into the mitochondria, a process mediated by tissue-specific isoforms of CPT1, the so-called liver (CPT1A), muscle (CPT1B), and brain (CPT1C) isoforms. It has been recently demonstrated that CPT1C is overexpressed in lung cancer (28). While 
A
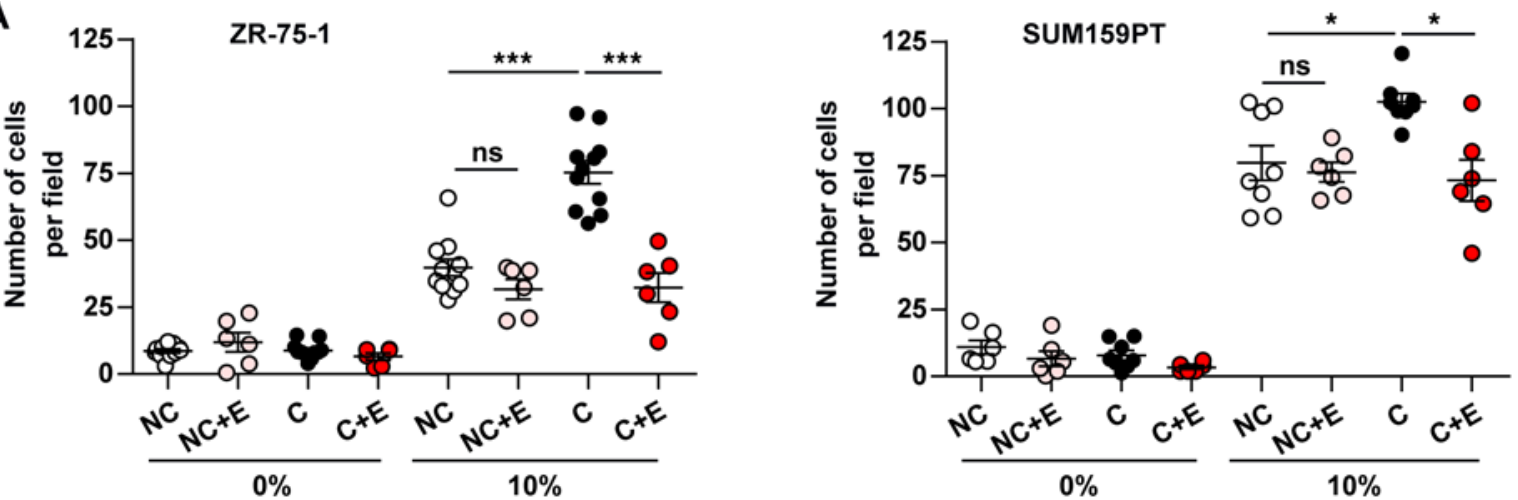

B
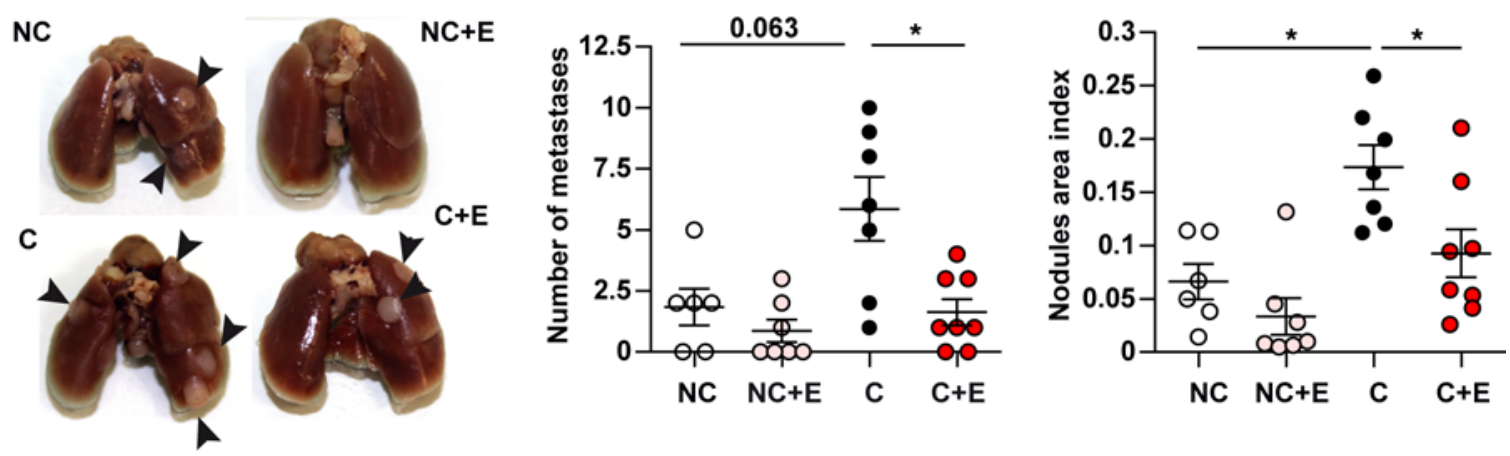

C
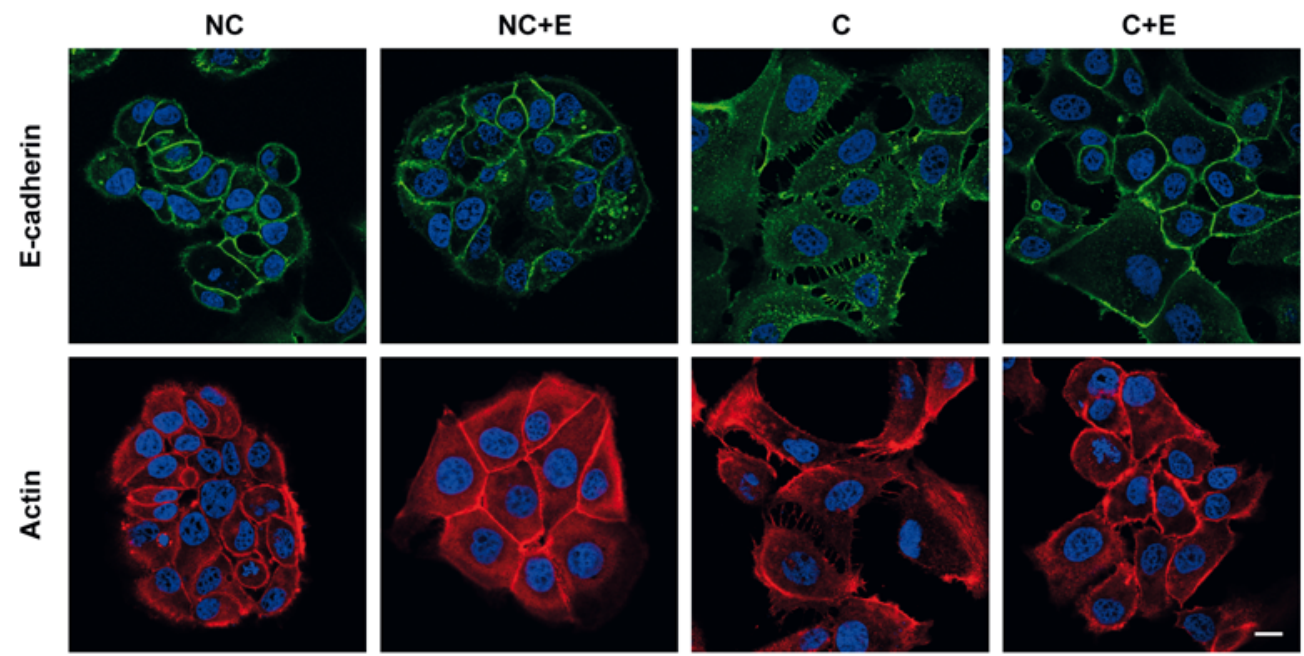

D

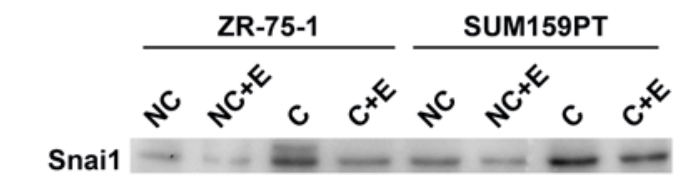
a-tubulin

Figure 4. The metabolic crosstalk between adipocytes and breast cancer cells contributes to epithelial to mesenchymal transition (EMT) and increases tumor invasion. (A) ZR-75-1 or SUM159PT cells were grown for 3 days on Transwells in the presence (C) or not (NC) of adipocytes and treated or not with Etomoxir (+E). After 3 days, cells were used for Matrigel invasion assays against medium containing either $0 \%$ or $10 \%$ FCS ( $n=6-11)$. (B) BALB/c mice were inoculated with murine breast cancer TS/A cells cultured in the presence or absence of adipocytes and treated or not with $30 \mu \mathrm{M}$ Etomoxir (+E) for 3 days prior to tail vein injection. Left, representative image of lungs from each group harvested at necropsy. The nodules present at the surface of the lungs are indicated by arrows. Right, quantification of the number and area of tumor nodules for each group of mice. NC, $n=6 ; N C+E, n=7 ; C, n=7 ; C+E, n=8$.

(C) ZR-75-1 cells were grown for 3 days on coverslips in inserts in the presence or not of mature adipocytes and treated or not with Etomoxir (+E). After 3 days, cells were fixed, and the indicated proteins were detected by immunofluorescence. Nuclei were labeled with DAPI. Scale bar: $20 \mu \mathrm{m}$. (D) Western blot of SNAI1 in ZR-75-1 and SUM159PT cells incubated in similar conditions. (C and D) At least 3 experiments were conducted, and representative experiments are shown. Bars and error flags represent means \pm SEM; statistically significant by Mann-Whitney $U$ test $(\mathbf{A}$ and $\mathbf{B}),{ }^{*} P<0.05,{ }^{* * *} P<0.001$. 
A

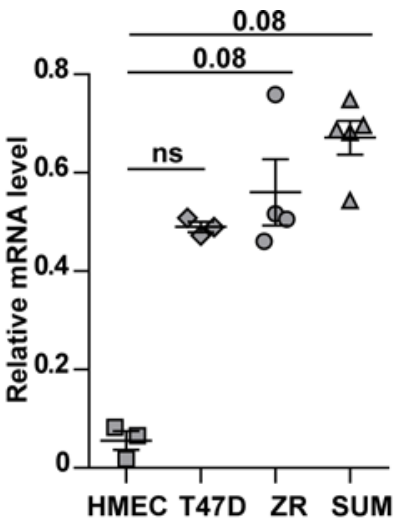

B

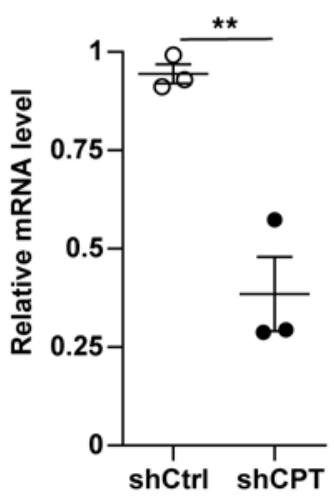

C

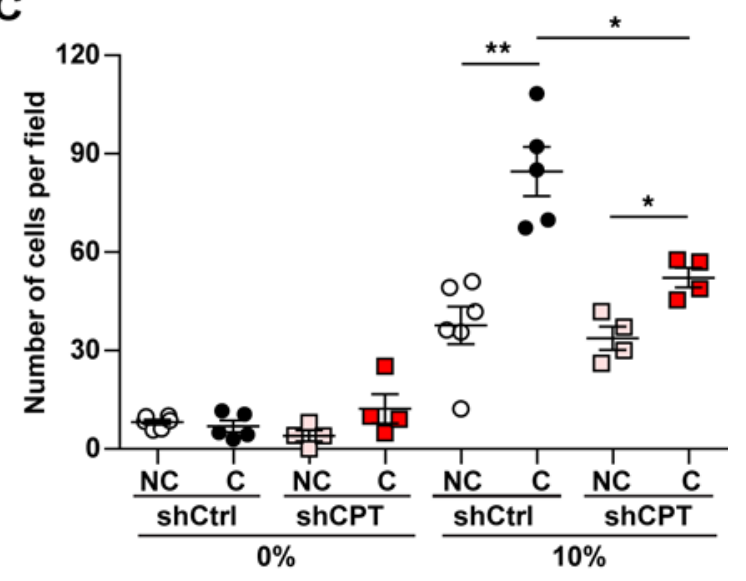

D

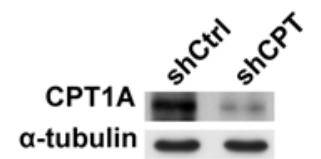

shCPT

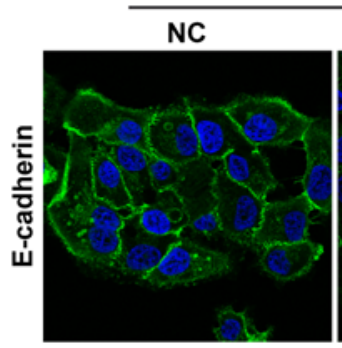

shCtrl
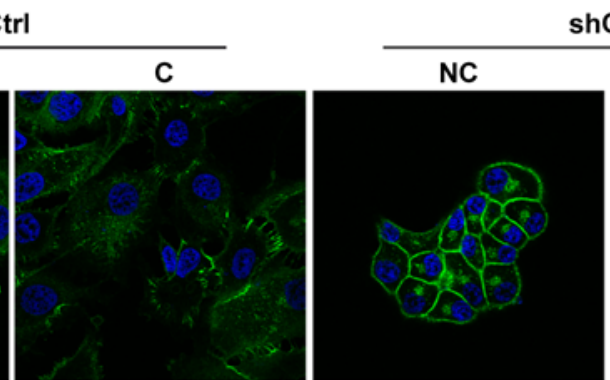

C
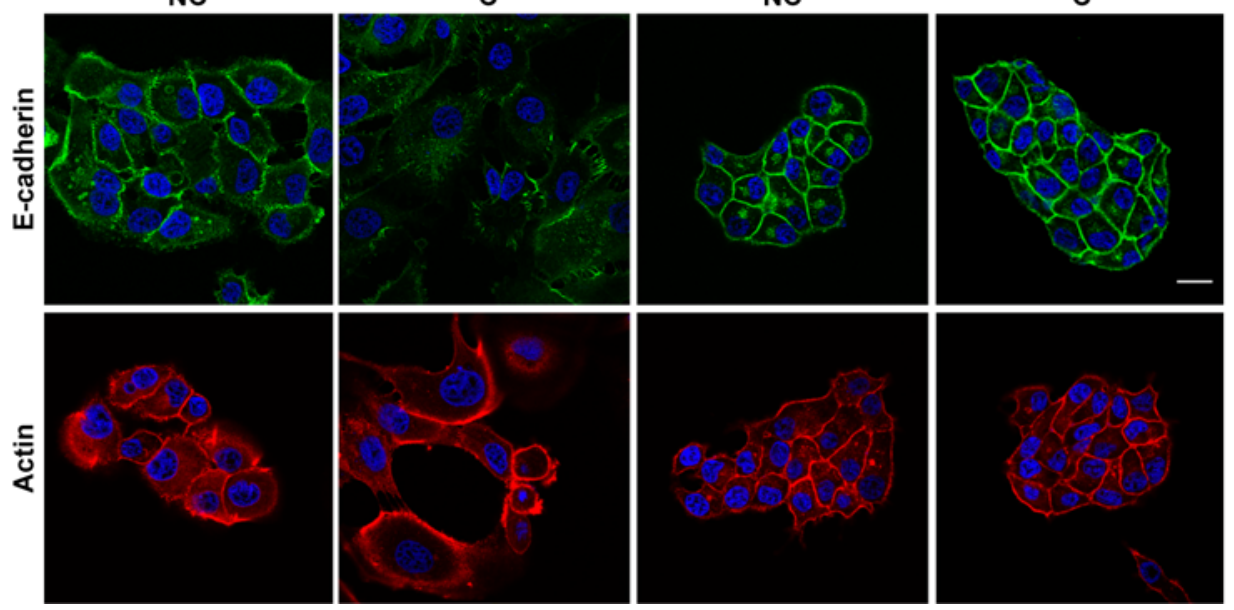

Figure 5. Downregulation of carnitine palmitoyltransferase 1A (CPT1A) expression in breast cancer cells inhibits the increased invasion and epithelial to mesenchymal transition (EMT) induced by adipocytes. (A) Upper panel: relative CPT1A mRNA expression in human mammary epithelial cells (HMEC) and in the indicated breast tumor cell lines (ZR: ZR-75-1; SUM: SUM159PT) ( $n=3-5)$. Lower panel: expression of CPT1A analyzed by immunoblot in ZR-75-1 cells cocultivated (C) or not (NC) with mature adipocytes for 3 days. (B) Quantification of the mRNA (upper panel, $n=3$ ) and protein levels (lower panel) of CPT1A in ZR-75-1 cells stably transfected with shControl (shCtrl) and shCPT1A (shCPT) vectors. (C) ZR-75-1 cells stably transfected with shCtrl and shCPT were grown on Transwells in the presence or not of mature adipocytes. After 3 days, cells were used for Matrigel invasion assays toward a medium containing either $0 \%$ or $10 \%$ FCS $(n=4-6)$. (D) Immunofluorescence staining, visualized by confocal microscopy, of E-cadherin (green) and Actin (red) in shControl and shCPT1A ZR-75-1 cells cocultivated or not with adipocytes for 3 days. Nuclei were labeled with DAPI. Scale bar: $20 \mu \mathrm{m}$. (A, B, and D) At least 3 experiments were conducted, and representative experiments are shown. Bars and error flags represent means \pm SEM; statistically significant by Mann-Whitney $U$ test ( $\mathbf{A}$ and $\mathbf{C}$ ) and Student's $t$ test $(\mathbf{B}),{ }^{*} P<0.05,{ }^{*} P<0.01$.

investigating the expression of CPT1 isoforms (CPT1A, -B, and -C) in breast cancer cell lines, we observed that only CPT1A was overexpressed in breast cancer as compared with normal breast cells (Figure 5A, upper panel). Similar results have been previously observed using different couples of tumor/normal cells (29). Interestingly, CPT1A expression was increased in cocultivated cells as shown for ZR-75-1 cells (Figure 5A, lower panel). In order to further validate the role of FAO in breast cancer aggressiveness, CPT1A stably silenced ZR-75-1 cells were engineered. CPT1A expression was efficiently inhibited by shRNA expression with a 75\% decrease in mRNA levels (upper panel) and a correlated decrease in protein levels (Figure 5B, lower panel). Before performing coculture experiments, we ensured that the growth rate of shCPT1A ZR-75-1 cell line was similar to control cells (Supplemental Figure 12A). In addition, after coculture or not with adipocytes, the number of viable 
cells was unchanged between shCPT1A and control cells (Supplemental Figure 12B). The absence of CPT1A expression specifically impaired the increase in tumor aggressiveness induced by adipocytes (Figure 5, C and D). In fact, a significant decrease in invasion was observed in cocultivated shCPT1A cells as compared with control cells (Figure 5C). In addition, cocultivated shCPT1A cells retained a characteristic epithelial morphology and formed compacted colonies with E-cadherin expressed at the cell membrane, similar to noncocultivated cells (Figure 5D), in contrast to cocultivated control cells that exhibit EMT. Therefore, our results, using both pharmacological inhibition and gene repression strategies, demonstrate that uncoupled FAO is a driving process in the increased cellular invasion and metastasis promoted by tumor-surrounding adipocytes.

ATGL is a key lipolytic enzyme involved in tumor aggressiveness that ensures FFA supply for enhanced FAO activity over time. Lipids stored as TG must be released as FFAs in order to fuel FAO to sustain invasion following the initial crosstalk with adipocytes; this process is important for circulating tumor cells that will secondarily reach distant organs. As shown in Figure 6A (left panel), we found that, when tumor cells were previously cocultivated for 3 days with adipocytes and then grown alone, a progressive decrease in TG content was observed during the first 48 hours before returning to the basal level of noncocultivated cells. This was associated with a significant increase in the levels of glycerol released into the culture medium (Figure 6A, right panel), highlighting the existence of a lipolytic process in these cells. The lipolysis process has been mainly studied in adipocytes where it is carried out by 3 major lipases, ATGL, HSL, and MAGL (16). Among these lipases, ATGL is the rate-limiting enzyme for the initiation of catabolism of TG in adipose and nonadipose tissues such as heart and muscle (30). We therefore investigated the expression of these lipases in either normal or tumoral breast epithelial cells with a wide range of aggressiveness from nonaggressive epithelial-like cells (ZR-75-1, MCF-7, and T47D) to highly aggressive fibroblast-like cells (SUM159PT, MDA-MB-231, and HTM-3522-T4-2). As shown in Figure 6B, ATGL expression was not detected in HMEC, and its level of expression was higher in aggressive cells as compared with nonaggressive cells. Similar results were obtained with HSL (with the exception of its low expression in HMEC cells), whereas monoacylglycerol lipase (MAGL) was expressed in nonaggressive and aggressive cells, with a slightly higher expression in the latter group (Figure 6B). The regulation of ATGL expression was observed in cocultivated cells, with the exception of the highly aggressive triple negative MDA-MB-231. Similar upregulation of HSL and MAGL expression was observed in nonaggressive cell lines; this effect was less pronounced for MAGL (Figure 6C). Due to its role in hydrolyzing TG, its pattern of expression, and its regulation by coculture, we decided to stably invalidate ATGL in ZR-75-1 cells. Hence, a marked decrease, higher than 80\%, was detected at protein levels in ATGL deficient cells (Figure 6D). The doubling time of these cells was unaffected by ATGL deficiency (Supplemental Figure 13A). Coculture had no effect on cell number in both control and shATGL cells (Supplemental Figure 13B). In cells previously cocultivated with adipocytes, and therefore loaded with lipids, we further demonstrated that lipolysis was strongly impaired in shATGL cells in contrast with control cells (Figure 6E). When invasion assays were performed in cocultivated cells, we found that the proinvasive effect of adipocytes was profoundly decreased in ATGL-deficient cells as compared with ATGL-proficient cells (Figure 6F). Accordingly, in the presence of adipocytes, the ATGL-deficient cells retained their epithelial-like aspect and E-cadherin continued to be expressed at the cell surface (Figure 6G). Taken together, these results show that ATGL plays a key role in regulating the lipid network, and subsequent invasiveness, of breast cancer cells cocultivated with adipocytes. We finally investigated whether this crosstalk exists in primary human tumors. As shown in Figure 7A, we consistently observed that breast cancer cells at the adipocyte/tumor interface contained lipid droplets. Given the great importance of ATGL expression and function in the adipose/ cancer crosstalk, and its regulation by coculture, we decided to study its expression in human tumors along with MAGL. A tumor-macroarray (TMA) of 69 patients exhibiting breast cancer as well as normal breast epithelial cells was used (Supplemental Table 1). As shown in Figure 7B, in accordance with the results obtained in cell lines, we observed that ATGL was only expressed in human breast cancer cells, whereas MAGL expression was detected both in normal and cancerous tissues. Importantly, ATGL expression in tumors was associated with aggressive features. In fact, a significant increase in ATGL expression was observed in tumors of high grade $(P<0.001)$ and in tumors expressing HER2 $(P=0.02$; Supplemental Table 2). By contrast, MAGL expression was not associated with poor prognosis factors in breast cancer (Supplemental Table 3). Another important finding of this IHC study was the fact that, when TMA contained adipose tissue, a highly significant increase of ATGL expression was observed in 
A

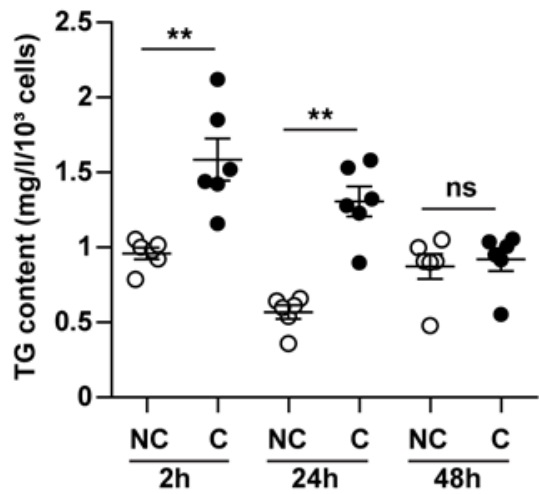

B

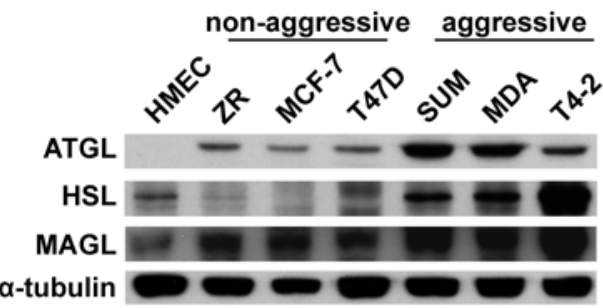

E

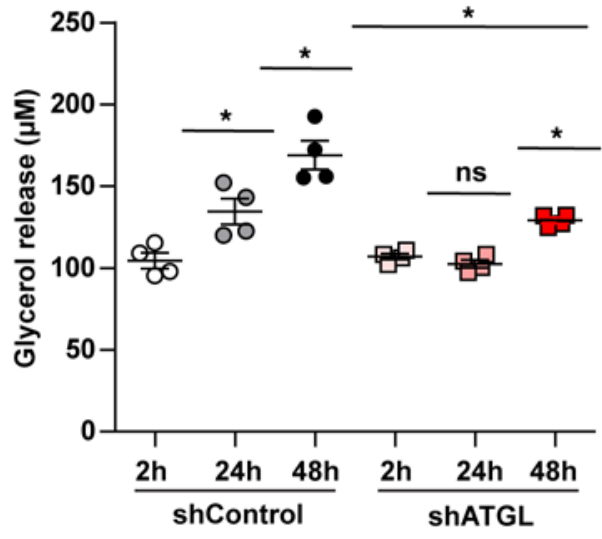

G

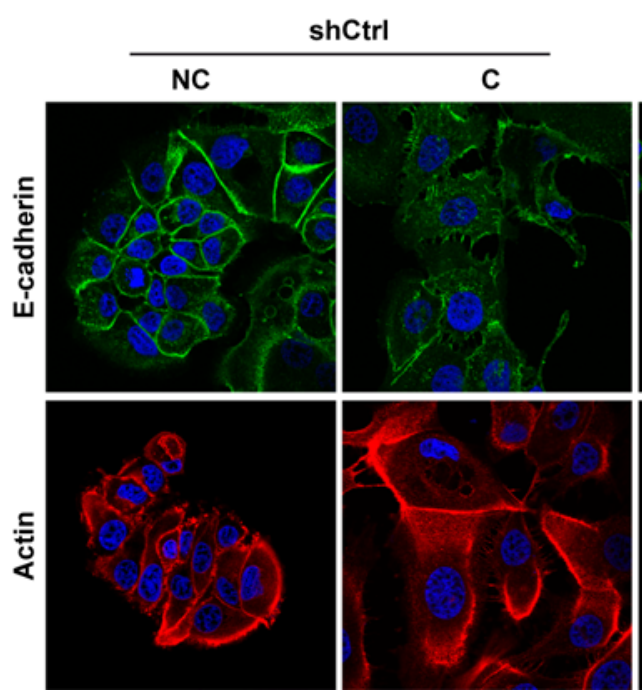

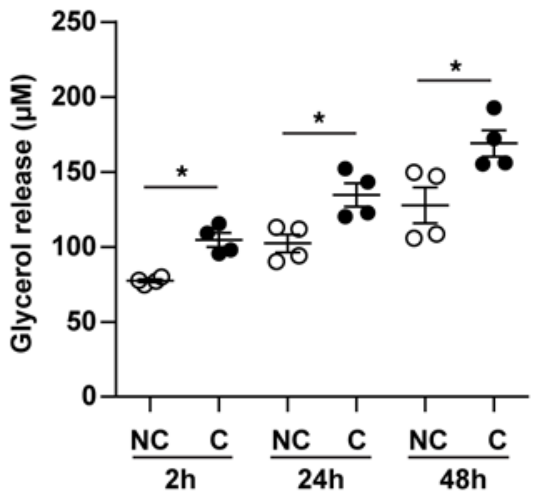

C

$$
\begin{aligned}
& \begin{array}{lrll}
\multicolumn{2}{c}{\text { non-aggressive }} & & \multicolumn{2}{c}{\text { aggressive }} \\
& \text { T47D } & \text { SUM MDA }
\end{array} \\
& \text { NC C NC C NC C NC C }
\end{aligned}
$$

ATGL

HSL

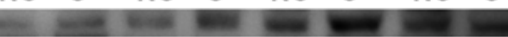

MAGL

$\beta$-actin
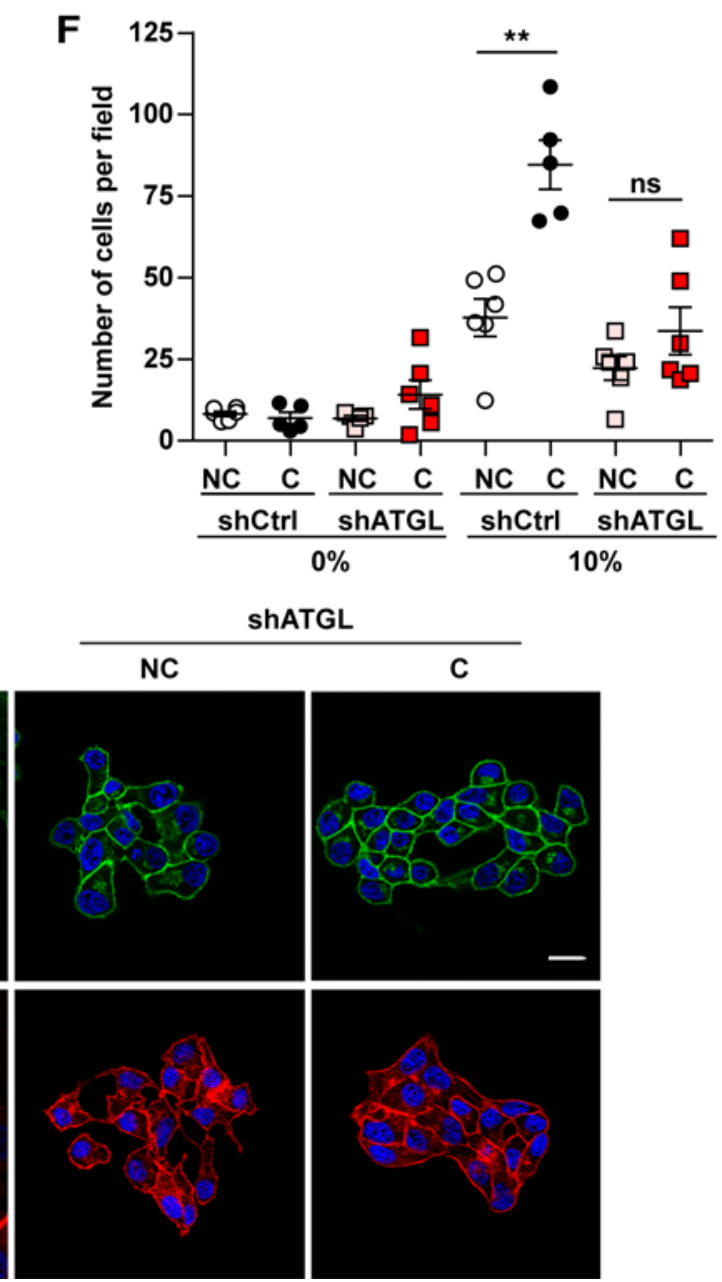

Figure 6. Breast cancer cells possess an adipose triglyceride lipase-dependent (ATCL-dependent) lipolytic pathway that favors cancer aggressiveness in the presence of adipocytes. (A) ZR-75-1 cells were cocultivated (C) or not (NC) with adipocytes for 3 days. Then, at indicated times after adipocyte removal, tumor cell TC content (left, $n=6$ ) and the glycerol release (right, $n=4$ ) were measured. (B) ATCL, HSL (Hormone-Sensitive Lipase), and MACL (monoa- 
cylglycerol lipase) protein levels were analyzed in human mammary epithelial cells (HMEC), nonaggressive (ZR, MCF-7, and T47D), and aggressive breast tumor cells (SUM, MDA, and T4-2) by Western blot (MDA, MDA-MB-231; T4-2, HMT-3522-T4-2). (C) Similar experiments performed in tumor cells cocultivated (C) or not (NC) with adipocytes for 3 days. (D) ATCL protein levels analyzed in ZR-75-1 cells transfected with shControl (shCtrl) or shATCL vectors. (E) Glycerol content measured in the medium of ZR-75-1 tumor cells transfected with shControl or ShATCL vectors and cocultivated in the presence of adipocytes for 3 days $(n=4)$. (F) ZR-75-1 cells stably transfected with shControl or shATCL were cocultivated or not with adipocytes. After 3 days, cells were used for matrigel invasion assays in a medium containing either $0 \%$ or $10 \%$ FCS $(n=5-6)$. (C) Staining of E-cadherin (green) and Actin (red) by immunofluorescence in these cells. Nuclei were labeled with DAPI. Scale bar: $20 \mu \mathrm{m}$. (B-D and G) At least 3 experiments were conducted, and representative experiments are shown. Bars and error flags represent means \pm SEM; statistically significant by Mann-Whitney $U$ test, ${ }^{*} P<0.05,{ }^{* *} P<0.01$.

tumor cells at close proximity to adipocytes in all the available samples (Figure 7B, right panel). Taken together, these results demonstrate that ATGL is specifically expressed by breast tumor cells in vitro and in vivo. Its function in cancer cells allows the liberation of FFAs stored in lipid droplets to sustain the proinvasive activity of adipocytes, and its striking regulation in human breast tumors highlights that this crosstalk between mature adipocytes and breast tumors does exist in vivo.

\section{Discussion}

Our study reveals a complex metabolic network favoring tumor invasion and metastasis established between breast cancer cells and surrounding adipocytes at the tumor invasive front. A metabolic symbiosis occurs between the two cell populations that favors the storage and mitochondrial use of adipocyte-derived FFAs by cancer cells. In order to sustain tumor cell invasion following the initial crosstalk with adipocytes, ATGL-dependent lipolytic activity allows the liberation of stored FFAs over time, a process probably key to distant metastasis (summarized in Figure 8).

The initiating event of this crosstalk is the ability of tumor cells to induce a lipolytic process in adipocytes. We have previously demonstrated that, in breast cancer, adipocytes present at the invasive front exhibit extensive phenotypical changes leading to their delipidation, decrease in size, and occurrence of an activated phenotype, becoming cells that we named CAAs (10). The delipidation of tumor-surrounding adipocytes is the result of at least 2 processes, including "dedifferentiation" resulting from the activation of the Wnt/ $\beta$-catenin pathway (13) and lipolysis (Figure 1C). The signal emanating from tumor cells that is responsible for the induction of lipolysis in adipocytes remains unidentified. In this study, we found that neither propranolol (a $\beta$-AR antagonist) nor UK14304 (an $\alpha 2$-AR agonist) were able to inhibit the lipolysis induced by tumor secretions, therefore eliminating the role of catecholamines in this process. Different results were obtained by Nieman et al., since propranolol partially reversed ovarian cancer cell-induced HSL activation (15), suggesting that this process might be regulated in a tumor-type-specific manner. The FFAs liberated by adipocytes are then taken up by tumor cells. In an adipocyte-rich microenvironment, constant FA import into tumor cells leads to accumulation of lipids as TGs within lipid droplets, as shown in vitro (Figure 1, A and B, and Supplemental Figure 1) and in vivo (Figure 7A). TG accumulation protects against FA-induced lipotoxicity as already described for nonadipose cells $(31,32)$, including cancer cells (32). Cancer cells that have accumulated lipids undergo extensive metabolic changes, including increased UCP2 and IF1 expression, which lead to uncoupled FAO, resulting in a fall in ATP content. This reduced ATP content initiates a circle that maintains this metabolic remodeling through activation of AMPK, leading to mitochondria biogenesis and inhibition of ACC to maintain FFA flux to the mitochondria (summarized in Figure 3E). How is this metabolic remodeling initiated? We hypothesize that the massive influx of cellular FFAs in breast cancer cells delivered by proximal adipocytes induces lipotoxicity and promotes, to counteract this lipotoxicity, the passive mitochondrial uncoupling, as extensively described in other contexts (33). In turn, increased expression of UCP2 (also upregulated by FFA influx), as well as upregulation of IF1, maintains this metabolic remodeling $(23,34)$.

Our results shed new light on the consequences of the metabolic symbiosis instilled between cancer cells and tumor-surrounding adipocytes in tumor progression. In fact, in our model, the transferred FFAs are used to perform uncoupled FAO, and this metabolic pathway controls cell invasion, although not proliferation or survival. These results are in contrast with those obtained by Nieman et al. in ovarian cancer (15), where increases in cell growth both in vitro and in vivo was highlighted as the key resulting effect of this crosstalk. It is conceivable that, in our model, the stronger increase in FAO than that observed in ovarian cancer (15) could lead to a reduction in FFAs available for use as membrane building blocks or signaling lipids, thus constraining cancer cell proliferation. Emerging evidence demonstrates that FAO is a more important metabolic pathway for tumor cells than initially thought (for review, see ref. 6). Our 

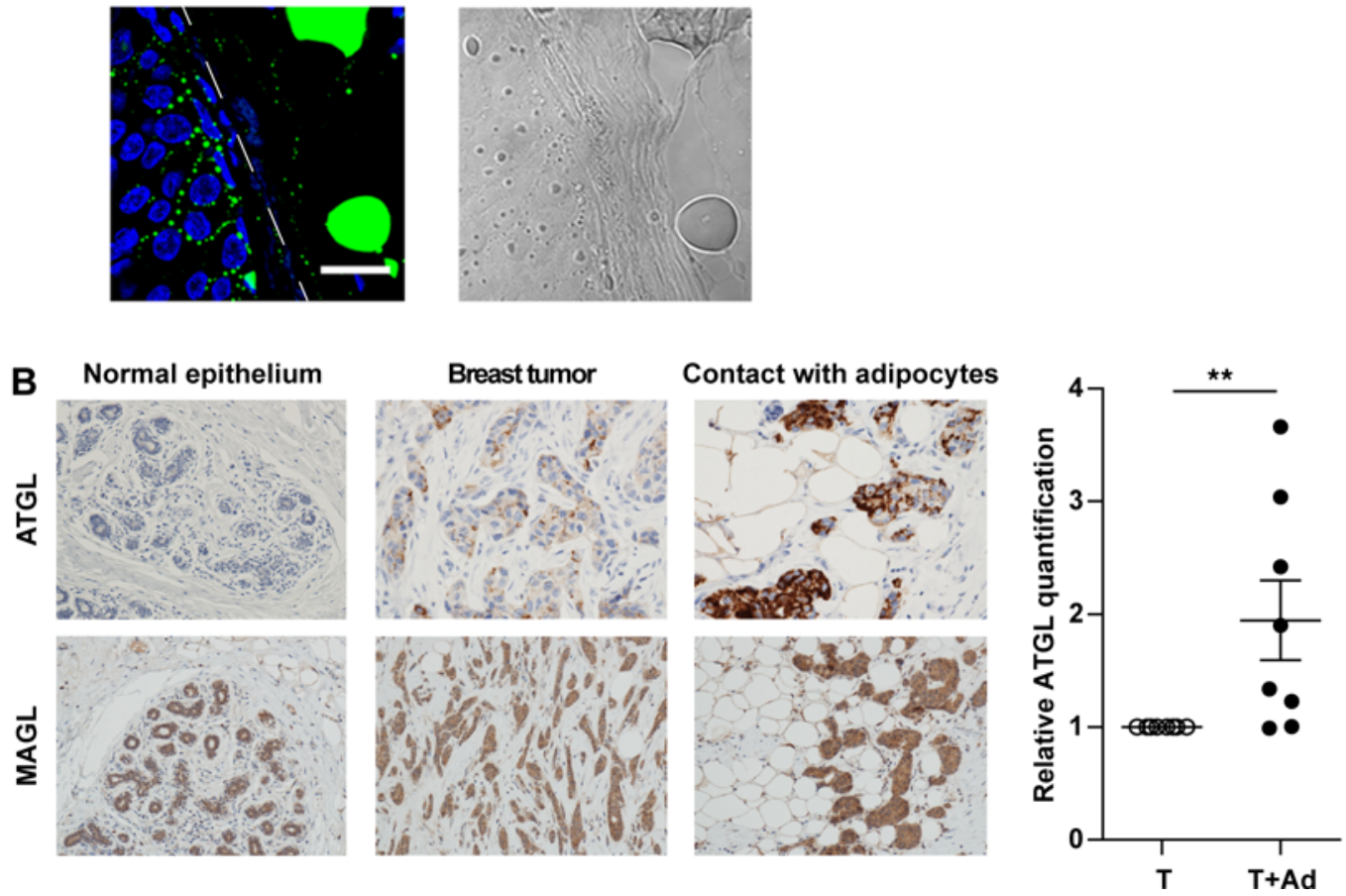

Figure 7. Breast cancer cells accumulate lipids and present upregulated adipose triglyceride lipase (ATGL) expression at close proximity to adipocytes. (A) Left, representative staining of lipid accumulation (detected by bodipy, in green) at the invasive front of a human breast tumor sample. The interface between breast cancer cells and adipocytes is indicated by a white dashed line. Scale bar: $20 \mu \mathrm{m}$. Nuclei were labeled with DAPI. Right, phase contrast light microscopy observation of the same sample. (B) Left, representative immunohistochemical staining of ATCL and MAGL (monoacylglycerol lipase; in brown) in human samples, with hematoxylin counter-staining. A representative staining obtained in normal breast epithelium (objective 20x), in breast tumors and in areas where breast tumors are in contact with adipocytes (objective 40x), is shown. Right, quantification of ATCL in human tumors where tumor cells are in contact with adipocytes $(T+A d)$ or not $(T)(n=8)$. Bars and error flags represent means $\pm \mathrm{SEM}$; statistically significant by Student's $t$ test, ${ }^{* *} P<0.01$.

work shows that the metabolic symbiosis between breast cancer cells and tumor-surrounding adipocytes leads to increased invasion both in vitro and in vivo and that this effect is supported by uncoupled FAO, eliminating the role of an increase in energy supply. In fact, its effect on tumor progression is interrupted by either pharmacological inhibition or downregulation of CPT1A, a key limiting actor in FFA transport into mitochondria (Figure 4 and 5). It is important to note that, in our study, the increased cellular invasion observed in cocultivated cells is not due to a "Warburg-like" effect, since the use of a glycolysis inhibitor (2DG) doesn't inhibit the cellular invasion induced by adipocytes (Supplemental Figure 10). A recent study demonstrated that ectopic expression of UCP2 in MCF7 breast cancer cells led to a decreased mitochondrial membrane potential and increased tumorigenic properties, as demonstrated by cell migration, invasion, and anchorage-independent growth (35). UCP2 appears to be overexpressed in human breast cancer, as well as in colon cancer with metastatic localization $(35,36)$. We further demonstrate that the resulting increased invasion observed in cocultivated cells is due to an EMT process, in accordance with our previous results (10), and we linked this EMT to uncoupled FAO, since both ETO treatment and CPT1A downregulation hamper this process. In accordance with our results, it has been demonstrated that, in normal cells, UCP2 has an important role in mediating TGF- $\beta 1$-induced EMT and kidney fibrosis (37). In addition to the proposed involvement of UCP2, a very interesting hypothesis emerging from recent studies is that intermediate metabolites such as acetyl-coA and NAD+, among others, contribute to epigenetic changes by regulating the activity of chromatin-modifying enzymes (for review, see ref. 38). This mechanism could therefore favor the EMT observed in our study. Indeed, the increase in FAO associated with decreased ETC activity observed in our model could contribute to the accumulation of such Kreb's cycle intermediates. Additional studies to explore these different hypotheses are clearly needed. 


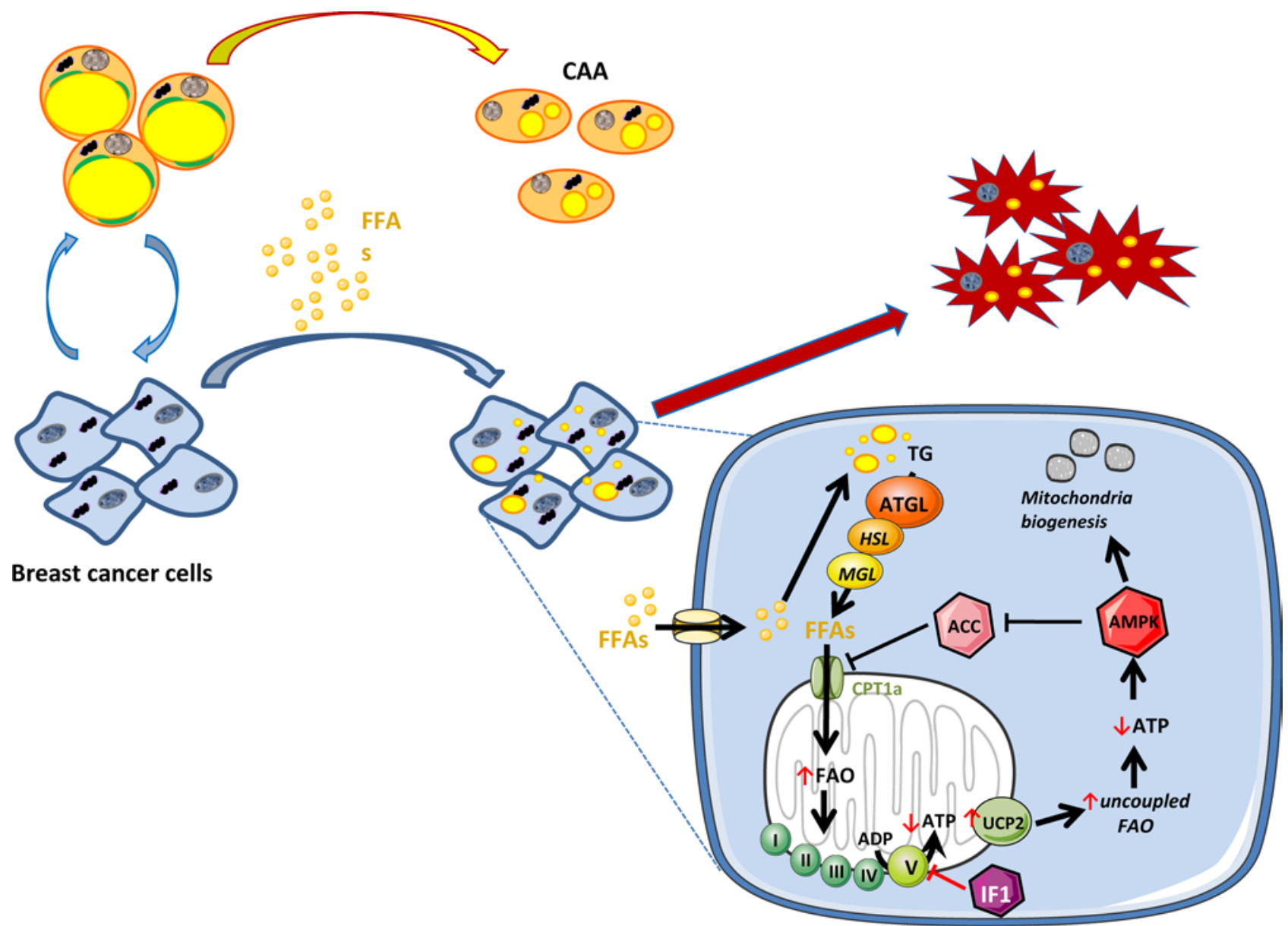

Figure 8. Tumor-surrounding adipocytes promote a metabolic remodeling in breast cancer cells, leading to increased aggressiveness. Tumor secretions induce a lipolytic process in surrounding adipocytes, which then become cancer-associated adipocytes (CAA). The large quantities of free fatty acids (FFAs) released after adipocyte activation are uptaken and stored as triglycerides (TC) by cancer cells. In these cells, adipose triglyceride lipase (ATCL) overexpression activates a lipolytic process, leading to the release of FFA from lipids droplets and their translocation into mitochondria via the carnitine palmitoyltransferase 1A (CPT1A). The massive influx of lipids induces uncoupled fatty acid oxidation (FAO), characterized by uncoupling protein 2 (UCP2) and inhibitory factor 1 (IF1) overexpression and decreased ATP levels. The reduced ATP content activates AMPK, which promotes both mitochondrial biogenesis and FFA uptake into mitochondria via the acetyl-CoA carboxylase (ACC) inhibition. This circle maintains the metabolic remodeling of tumor cells and increases their aggressiveness.

One of the important findings in our study is the identification of ATGL as a major actor in this metabolic symbiosis that allows cancer cells to use, when needed, stored lipids to liberate FFAs. After the initial contact with adipocytes that loads cancer cells with lipids, the ability of tumor cells to progressively liberate FFAs in order to sustain invasive activities will be key for circulating tumor cells to reach distant organs. It is now acknowledged that activation of de novo FA synthesis is required for carcinogenesis (39). However, emerging evidences suggest that cancer cells can also rely on lipolytic pathways to use, when needed, stored lipids to acquire FFAs (39). In this regard, it has been demonstrated that FFAs liberated from lipid droplets are efficiently used to cope with the energy demand of nutritionally stressed cells (40). Concomitant TG synthesis and hydrolysis, such as observed in our study, has been described in both normal and pathological conditions (41-43). For example, in cardiac myocytes, the majority of lipids oxidized by mitochondria are first esterified into TG, then hydrolyzed before oxidation (41). In this study, overexpression of PPARa (which is also observed in our study) was shown to increase TG turnover, as well the expression of enzymes involved in TG synthesis (e.g., gpam, agpat, and dgat1) (41). In cancer cells, Nomura et al. (42) have shown that newly synthesized FFAs are immediately converted into neutral lipid stores and that the use of FFAs is dependent on their release. To our knowledge, our study is the first to show that ATGL, expressed in both human breast cancer cell lines and in human breast tumors, functions as a key enzyme in TG hydrolysis and that its expression is related to tumor aggressiveness, clinically highlighted by its significant overexpression 
in tumors of high grade (Supplemental Table 2). Our results also emphasize that ATGL is overexpressed in tumors expressing HER2 (Supplemental Table 2). HER2 oncogene overexpression specifically triggers redundant signaling cascades to increase the expression of all the major enzymes involved in de novo FA synthesis, as well as in lipid storage (44). Therefore, our results strongly suggest that ATGL, by providing a complementary lipolytic pathway to lipogenesis, offers a double-edged sword to fully promote the aggressive phenotype of these cells. Altogether, our results highlight that increased FFA availability and storage induced by oncogenic signals and microenvironmental changes required paired lipolytic activity to promote tumor progression. Our results are in apparent contradiction with the work of Nomura et al., which shows that MAGL is highly expressed in aggressive human cancer cells where it regulates a FA network enriched in oncogenic signaling lipids that promote tumor progression (42). In breast cancer, both in vitro and in vivo, we found only a slight difference in MAGL expression between normal epithelial cells and in nonaggressive and aggressive tumors, in contrast to their data, which was limited for breast tumors to the in vitro analysis of two cell lines (MCF-7 and 231MFP) (42). Therefore, our results suggest that, in breast cancer, this enzyme might be less important for tumor progression than in melanoma or ovarian cancer (42). It is now clearly emerging that tumor lipid metabolism is regulated not only by genetic and epigenetic changes in the tumor cells, but also by the availability of lipids provided by tumor-surrounding adipocytes. The different molecular steps identified in our work governing this complex metabolic symbiosis, and more specifically ATGL, may provide a therapeutic target to effectively impede breast cancer progression.

\section{Methods}

Supplemental Methods are available online with this article.

Cell lines and reagents. The murine 3T3-F442A preadipocyte line (provided by Philippe Valet, I2MC, Toulouse, France) was cultured in DMEM medium supplemented with $10 \%$ fetal calf serum (FCS). Differentiation was induced by incubating confluent cells in differentiation medium (DMEM 10\% FCS and $50 \mathrm{nM}$ insulin) for up to 14 days as described previously (45). HMEC (provided by Jacques Piette, IGMM, Montpellier, France) were grown in MEBM medium (Lonza), containing transferrin $(5 \mu \mathrm{g} / \mathrm{ml})$ and isoproterenol $\left(1 \times 10^{-5} \mathrm{M}\right)$. The human breast cancer line ZR-75-1 (Sigma-Aldrich) was cultured in DMEM medium 10\% FCS, and the SUM159PT line (provided by Jacques Piette, IGMM, Montpellier, France) was grown in Ham F12 (50:50) medium complemented with 5\% FCS, $1 \mu \mathrm{g} / \mathrm{ml}$ hydrocortisone (Sigma-Aldrich, Saint-Quentin les Ulysses, France) and 0.2 UI/ml insulin. HMT-3522-T4-2, a malignant breast cancer line derived from human mammary epithelium (46) (provided by Emma Liaudet-Coopman, IRCM, Montpellier, France) was cultured in H14 medium (47). The human breast cancer lines MCF-7, T47D, and MDAMB-231 (provided by Kerstin Bystricky, LBME, Toulouse, France) and the murine breast cancer line TS/A (provided by Philippe Valet, I2MC, Toulouse, France) were cultured in RPMI 1640 medium 10\% FCS. All the lines were maintained at $37^{\circ} \mathrm{C}$ in a humidified atmosphere with $5 \% \mathrm{CO}_{2}$ and used within 2 months after resuscitation of frozen aliquots. The suppliers, references, and dilutions used for antibodies are listed in Supplemental Table 4. DMSO, Oil Red O, Toluidine Blue O, and Etomoxir (used at 1-30 $\mu \mathrm{M}$ final concentration) were purchased from Sigma-Aldrich. The bodipy lipid probe (used at $1 \mu \mathrm{g} / \mathrm{ml}$ ) and DAPI (used at $1 \mu \mathrm{M}$ final concentration) were obtained from Invitrogen.

RNA interference studies. To stably switch off ATGL and CPT1A gene expression in ZR-75-1 cells, we used replicative shRNA-expressing vectors (pEBVsiRNA), which impose a very strong and stable gene silencing in human cells, even after several months in culture. siRNA design was performed using the DSIR program (48). RNAi sequences against ATGL stretched from nucleotides 11-29 (with ATG as the first nucleotide, GCGAGAAGACGTGGAACAT; pBD2338) and from nucleotides 203-221 against CPT1A (GCGTGATGACAACGATGTA, pBD2405 or pBD2408). pEBVsiRNA vectors were transfected into ZR-75-1 cells using Lipofectamine, and knocked-down cell populations were selected with $30 \mu \mathrm{g} / \mathrm{ml}$ of hygromycin B (Invitrogen). ATGL and CPT1A silenced cells were termed as shATGL and shCPT1A cells, respectively. As a control, we used the pBD650 plasmid, which expresses an inefficient shRNA sequence (49).

Coculture, cell proliferation, and invasion assays. Coculture and invasion assays were conducted as previously described (10). Briefly, $5 \times 10^{4}$ (SUM159PT and MDA-MB-231) or $1 \times 10^{5}$ (ZR-75-1 and T47D) cells were seeded in the top chamber of the Transwell system $(0.4 \mu \mathrm{M}$ pore size $)$ and cocultivated with or without adipocytes in the bottom chamber. Similar experiments were performed with shCtrl, shATGL, or shCPT1A ZR-75-1 cells. In indicated experiments, cells were treated or not during coculture with Etomoxir 
for 3 days. For viable cell number evaluation, after coculture in the presence or not of adipocytes, tumor cells were fixed with methanol, stained with Toluidine Blue O buffer ( $1 \%$ supplemented with $0.1 \mathrm{M}$ Borax), washed several times with water, air-dried, and dissolved in lysis buffer (6.25 mM Tris-HCL (pH 6.8), 10\% glycerol, $2 \%$ SDS, 10\% $\beta$-mercaptoethanol; Sigma-Aldrich). The quantification of the absorbance (measured at $570 \mathrm{~nm}$ ) was performed in duplicate. Matrigel invasion assays were conducted as described (10).

Oil Red $O$ and bodipy staining. The intracellular lipid content was evaluated with Oil Red O or Bodipy 493/503. For Oil Red O staining, cells in culture were fixed in 3.7\% paraformaldehyde for 20 minutes at room temperature (RT), rinsed twice with water, and air-dried. The cells were then stained for 20 minutes with Oil Red $\mathrm{O}$ in $60 \%$ (v/v) isopropanol and washed. For bodipy staining, after fixation, cells were permeabilized with PBS $0.2 \%$ TritonX-100 for 5 minutes and incubated (30 minutes) with Bodipy and DAPI (5 minutes). After washes, cells were mounted in Vectashield medium (Vactor Laboratories). Fluorescence images were acquired with a confocal laser microscopy system (Confocal TIRF Olympus FV1000). The image resolution was $512 \times 512$ pixels and scan rate was $400 \mathrm{~Hz}$.

$T G$ content analysis. Tumor cells were grown for 3 days in the presence or not of adipocytes. At the end of coculture, tumor cells were resuspended in $15 \mu \mathrm{l}$ of TG buffer (10 mM Tris-HCL (pH 7.5), 1 mM EDTA). TG content was quantified using a colorimetric kit (Biomerieux). Results were read at a wavelength of $505 \mathrm{~nm}$.

Measure of lipolysis. HMEC and tumor cell CM containing 0.2\% delipidated BSA (Sigma-Aldrich) were collected after 12 hours without serum. Glycerol released into adipocytes medium, incubated or not in the presence of CM from the indicated lines for 3 days, was measured using the Glycerol-Free Reagent Kit (Sigma-Aldrich). In certain experiments, shCtrl or shATGL ZR-75-1 tumor cells were cocultivated with adipocytes for 3 days and then grown alone. The measure of glycerol released was performed at the indicated time after coculture end.

Transfer of radioactive lipids between mature adipocytes and tumor cells. Preadipocyte 3T3-F442A cells at confluence were incubated in differentiation medium in the presence of $0.12 \mu \mathrm{Ci} / \mathrm{ml}$ radiolabeled palmitate acid. After 11 days, the human breast cancer cell line ZR-75-1 was seeded in the upper chamber of the Transwell system for coculture or not with radiolabeled adipocytes for 3 and 5 days in a medium without radiolabeled palmitate acid. ZR-75-1 and adipocytes were washed and then suspended in $500 \mu 1$ of lysis buffer. The radioactivity was counted.

Measure of FAO. HMEC, ZR-75-1, and SUM159PT cultivated alone or with adipocytes for 3 days were incubated with $2 \mathrm{ml}$ of warmed $\left(37^{\circ} \mathrm{C}\right)$, pregazed $\left(95 \% \mathrm{O}_{2}-5 \% \mathrm{CO}_{2}, \mathrm{pH} 7.4\right)$, modified Krebs-Henseleit buffer containing $1.5 \%$ FA-free BSA, $5 \mathrm{mM}$ glucose, $1 \mathrm{mM}$ palmitate, and $0.5 \mu \mathrm{Ci} / \mathrm{ml}\left({ }^{14} \mathrm{C}\right.$ ) palmitate (Perkin Elmer) for 60 minutes. Cells were then collected in $800 \mu \mathrm{l}$ of lysis buffer. A microtube containing $300 \mu \mathrm{l}$ of benzethonium hydroxide (Sigma-Aldrich) was placed in the vial to capture the ${ }^{14} \mathrm{CO}_{2}$. The buffer was acidified ( $1 \mathrm{ml}$ of $1 \mathrm{M} \mathrm{H}_{2} \mathrm{SO}_{4}$ ), and the vial was sealed. After 120 minutes, the radioactivity was counted (complete oxidation) (CytoScint, MP Biomedicals). Homogenate $(500 \mathrm{ml})$ was placed into glass tubes to extract lipids with chloroform-ethanol $(2: 1)$ and $2 \mathrm{M} \mathrm{KCl}-\mathrm{HCl}$. After centrifugation, the aqueous phase was quantified (incomplete oxidation). C2C12 was used as a positive control. All assays were performed in triplicates. Data were normalized to protein content.

$R N A$ extraction and quantitative PCR. Total RNAs were extracted using the RNeasy mini kit (Qiagen). Gene expression was analyzed using real-time PCR as described (50). Forward and reverse primers sequences are listed in Supplemental Table 5. 18S ribosomal, HPRT, or GAPDH RNA were used to normalize gene expression. Oligonucleotide primers were designed using the Primer Express software (PerkinElmer) as previously described (50).

Mitochondrial DNA analysis. Total DNA was extracted (DNeasy, QIAGEN), and the quantity of mitochondrial DNA was evaluated by quantitative PCR (qPCR) as previously described (51). Results were presented as a ratio between mitochondrial DNA (cytochrome c subunit, COX1) and genomic DNA (cyclophilin A and $Y W H A Z$ ). Oligonucleotide sequences are listed in Supplemental Table 5.

Transmission electron microscopy. $1 \times 10^{5}$ cells/well of ZR-75-1 were seeded in the upper chamber of the Transwell system for coculture with or without adipocytes for 4 days. Cells were fixed in $2 \%$ glutaraldehyde, $3 \%$ paraformaldehyde in $0.1 \mathrm{M}$ (pH 7.4) cacodylate buffer, and treated with $1 \%$ osmium tetraoxide. Specimens were rinsed with water and embedded in $0.5 \%$ uranyl acetate. After dehydration in ethanol gradients, cells were embedded in Epon812 resin (Delta-Microscopies). Tissue sections of $80 \mathrm{~nm}$ were cut (Ultracut Reichert ultramicrotome) and placed on copper grids, stained with uranyl acetate and lead citrate, and examined in a Jeol JEM-1400 Transmission electron microscope coupled to a digital camera (Gatan Orius, Gatan Inc.). 
ATP content and percent of glycolytic ATP. Global ATP content and proportion of glycolytic ATP were determined using CellTiter Glo luciferin-luciferase assay (Promega) and specific inhibitors of glycolysis and OXPHOS (iodoacetate and oligomycin, respectively). After coculture, tumor cells were seeded in 96-well plates (5,000 cells/well) and incubated for 18 hours. Cells were then stimulated for 1 hour in coculture or KHB medium with specific inhibitors. Iodoacetate and oligomycin, (Sigma-Aldrich), were used at $100 \mu \mathrm{M}$ and $10 \mu \mathrm{g} / \mathrm{ml}$, respectively. Luminescence was measured by using Luminoskan Ascent (Thermo Electron Corporation) microplate luminometer. The glycolytic proportion was calculated with this formula: (control - Iodoacetate) $\times 100 /$ (control - [Iodoacetate and oligomycin]).

Lactate dosage. Lactate released into the medium by tumor cells, grown alone or in the presence of adipocytes for 3 days, was measured in $5 \mu 1$ aliquots using the Lactate FS kit (DiaSys Diagnostic Systems). TruCal U, provided in the kit, was used as a calibrator.

$O C R$ and ECAR. OCR and ECAR were measured using the XF24 analyzer (Seahorse Bioscience). After coculture or not, cells were seeded in 24-well XF24 cell culture plates at a density of 20,000 cells/ well for 18 hours in CM (simple culture or coculture). Media was then removed, and wells were washed and incubated for 1 hour at $37^{\circ} \mathrm{C}$ without $\mathrm{CO}_{2}$ in XF modified DMEM assay medium (Seahorse Bioscience) at $\mathrm{pH} 7.4$, supplemented with $1 \mathrm{mM}$ glutamine (glycolysis and mitochondrial stress tests), 2.5 mM glucose, $1 \mathrm{mM}$ sodium pyruvate, $0.5 \mathrm{mM}$ carnitine, and $1 \mathrm{mM}$ palmitate complexed with $0.2 \mathrm{mM}$ BSA (mitochondrial stress tests). For glycolytic tests, OCR and ECAR were measured in the basal state (no glucose) or after injection of $10 \mathrm{mM}$ glucose, $5 \mu \mathrm{M}$ oligomycin, and $50 \mathrm{mM}$ 2DG (Sigma-Aldrich). For mitochondrial stress tests, OCR was measured in the basal state $(1 \mathrm{mM}$ palmitate complexed with $0.2 \mathrm{mM}$ BSA) or after injection of $5 \mu \mathrm{M}$ oligomycin, $1 \mu \mathrm{M}$ FCCP (2-[2-[4-(trifluoromethoxy)phenyl] hydrazinylidene]-propanedinitrile), and rotenone with antimycin A (both at $5 \mu \mathrm{M}$ ). The proportion of coupled respiration (OCR basal-OCR oligomycin), proton leaks (OCR oligomycin-OCR rotenone/ antimycin), and nonmitochondrial respiration (OCR rotenone/antimycin) was calculated as previously proposed (21). After Seahorse Bioscience experiments, proteins were quantified to normalize results.

Western blot analysis. Total proteins were extracted as described previously (52). Proteins (50 $\mu$ g) were electrophoresed on SDS-PAGE and transferred onto PVDF membranes. After being blocked with $5 \%$ skimmed milk in Tris-buffered saline (TBS $1 \times$ ), the membranes were incubated with the appropriate primary antibodies. Then, the membranes were washed with $0.1 \%$ TBS-T and incubated with HRP-conjugated secondary antibodies (Santa Cruz Biotechnology Inc.). The immunoreactive protein bands were detected by enhanced chemoluminescence (ECL).

Animal studies. Twelve-week-old male syngeneic BALB/cJ host mice (Janvier Labs) with an average body weight of $22 \mathrm{~g}$ were used in this study. Mice were housed conventionally in an animal room at constant temperature $\left(20^{\circ} \mathrm{C}-22^{\circ} \mathrm{C}\right)$ and humidity $(50 \%-60 \%)$, and with a 12-hour light/dark cycle. All the mice had free access to food and water throughout the experiment. A catheter was indwelled into the femoral vein under anesthesia (sodium pentobarbital, 1:10; $0.2 \mathrm{ml}$ per mice; Ceva), sealed under the back skin, and glued on the top of the skull. The mice were housed individually and allowed to recover for 4 days. Then, a total of $1 \times 10^{5}$ TS/A cells, previously cocultivated or not for 4 days with mature adipocytes in the presence or not of Etomoxir $(30 \mu \mathrm{m})$, were injected through the catheter in mice. All the mice received the cell injection in less than 1.5 hours. Mice were sacrificed after 25 days, the lungs were immediately excised, and the number of tumor nodules on the lung surface was recorded. The mean number of nodules per lung area and the mean nodule area as a function of lung area (that defined the nodule area index) were measured by ImageJ software program.

Immunofluorescence. The breast tumor cell line grown on coverslips were cocultivated or not with adipocytes and treated or not with Etomoxir $(30 \mu \mathrm{M})$ for 3 days. Similar experiments were performed with shCtrl, shATGL, or shCPT1A cells. The coverslips were fixed in 3.7\% paraformaldehyde for 20 minutes at RT. After blocking (10\% FCS and 2\% BSA in PBS) for 1 hour, cells were incubated for 2 hours at RT with antibodies directed against E-cadherin (1:50) or with rhodamine-phalloidin (1:200). For E-cadherin staining, Alexa fluor 488 conjugated anti-mouse secondary antibody (1:400) was used for 30 minutes at RT. Coverslips were examined using an Olympus FV-1000 with 60× oil PLAPON OSC objective or a Zeiss LSM 710 confocal microscope with 40× oil PlanApo objective. A minimum of 3 independent experiments were performed. Images were processed to filter the noise with Fiji software, and a similar filter was used to analyze all acquisitions for the experiment.

Breast cancer tissue microarrays. A TMA developed by the Pathology Department of Toulouse Rangueil Hospital, containing specimens of breast cancer ( $n=69$ in duplicates) and normal epithelium ( $n=10$ in duplicates) was used to report ATGL and MAGL expression in breast cancer. All patients included in the 
study had localized disease without metastasis (as assessed by clinical and radiological examinations) at the time of the surgery. The surgery specimen was treated within 15 minutes of its removal to limit the delay between devascularization and freezing, thus ensuring the preservation of labile molecules. Tissue samples were placed in cryovials, frozen in liquid nitrogen, and stored at $-80^{\circ} \mathrm{C}$. One pathologist (Ghislaine Escourrou), who was blind to clinical data, independently selected the areas of interest used for the preparation of TMA. Selected tumor areas had a morphology and histological differentiation similar to the tumor. After selecting the sample block, $0.2-\mathrm{mm}$ diameter core samples were included according to a predetermined pattern in a recipient block.

IHC. Immunohistochemical experiments were performed to detect the expression of ATGL and MAGL in TMA. Immunostaining was performed with the EnVision FLEX Mini Kit, High pH (Dako Autostainer/Autostainer Plus). The TMA were immersed in xylene to remove paraffin and then rehydrated by successive baths of graded alcohol (100\%-70\% and then distilled water), followed by treatment with the antigen unmasking solution for antigen retrieving (Citrate Target Retrieval solution, Dako) in a water bath at $95^{\circ} \mathrm{C}$. After a saturation step by endogenous peroxidases (peroxidase blocking solution, Dako), the samples were incubated with the primary antibodies (diluted to 1/100). TMAs were then incubated with biotinylated and streptavidin-HRP conjugate secondary antibody. The samples were rinsed, treated with liquid DAB (BioGenex), and washed with water. Finally, the counter-staining was carried out with hematoxylin (Dako), and the various TMA were mounted with Eukitt reagent. The slides were digitally scanned via Hamamatsu Nanozoomer 2.0RS and analyzed with the device software provided by the manufacturer. Two pathologists, who were blind to clinical data, independently scored ATGL and MAGL expression in human tumors as negative, low, moderate (mid), or high. The intensity of ATGL staining in breast cancer cells in contact or not with adipocytes has been quantified by computer through the use of ImageJ software, with tumor staining separated using a deconvolution plug-in, as described in ref. 53.

Statistics. The statistical significance of differences between means was evaluated with unpaired Student $t$ tests when comparing only 2 groups. Student's $t$ test was 2 tailed. Multiple comparisons between groups were performed using Mann-Whitney $U$ test. The Benjamini-Hochberg procedure was applied for multiple comparisons. All reported $P$ values were 2 sided. For all statistical tests, differences were considered significant at the $5 \%$ level. Statistical analysis was performed using R 3.2 .2 software. $P$ values below $0.05\left({ }^{*}\right)$, $<0.01\left(^{* *}\right)$, and $<0.001(* * *)$ were deemed as significant.

Study approval. All animal experiments were performed in accordance with National Institute of Medical Research (INSERM) principles and guidelines. All experiments have been approved by the MidiPyrénées animal ethics committee (protocol number 07/858/03/07). Concerning the analysis of breast tumors by IHC, all patients signed a consent form for the use of their tissue samples for scientific purposes before surgical intervention.

\section{Author contributions}

YYW, LN, PV, and CM participated in designing research studies. YYW, CA, DM, BD, SD, IL, VL, NA, SLG, AG, CH, GE, and LN participated in conducting experiments and acquiring and analyzing data. JG supervised the statistical analysis. DB participated in providing plasmids for knockdown experiments. YYW, CA, DM, BD, NA, F. Bost, GSR, F. Bono, LN, PV, and CM participated in interpreting data. YYW, $\mathrm{CA}, \mathrm{LN}, \mathrm{PV}$, and CM participated in writing the manuscript. DM, NA, DB, FB, and MP participated in editing the manuscript. CM supervised the study.

\section{Acknowledgments}

Studies performed in our laboratories were supported by the French National Cancer Institute (INCA PL 2010-214 to PV and CM, INCA PL-2013-66 to PV and CM), the Ligue Régionale Midi-Pyrénées contre le Cancer (to CM), and the Fondation de France (to PV and CM). YYW is a recipient of a PhD fellowship from the Chinese Research Council. Both VL and IL received a $\mathrm{PhD}$ fellowship from ARC (Association Contre le Cancer). We acknowledge the Anexplo Phenotypage platform (INSERM US006), the staff of animal facilities for their expertise, and the cellular physiology and metabolomics core facility of CRCHUM and Montreal Diabetes Research Center for providing the Seahorse analyzer. We also acknowledge Pascale Belenguer and Serge Mazere for JC-1 experiments. This work also benefited from the (TRI)-RIO Optical Imaging Platform at Institut de Pharmacologie et Biologie Structurale (Genotoul, Toulouse, France) supported by grants from the Région Midi-Pyrénées (contrat de projets état-région), 
the Grand Toulouse community, ARC (Equipement 8505), CNRS, and the European Union through the Fonds Européen de Développement Régional program. The authors would also like to thank Emily Clement for the English editing of the manuscript.

Address correspondence to: Catherine Muller, IPBS CNRS UMR 5089, 205 route de Narbonne, 31077 Toulouse, France. Phone: 33.561.17.59.32; E-mail: muller@ipbs.fr. Or to: Laurence Nieto, IPBS CNRS UMR 5089, 205 route de Narbonne, 31077 Toulouse. Phone: 33-561-17-55-09; E-mail: Laurence.Nieto@ipbs.fr. Or to: Philippe Valet, UMR INSERM-UPS U1048, 1 Ave. du Pr Jean Poulhès Bâtiment L4 - BP 8422531432 Toulouse Cedex 4. Phone: 33-561-32-56-34; E-mail: Philippe.Valet@inserm.fr.

YYW's present address is: Department of Endocrine and Breast Surgery, The First Affiliated Hospital of Chongqing Medical University, Chongqing, China.

CA's present address is: Departments of Nutrition and Biochemistry and Montreal Diabetes Research Center, CRCHUM and Université de Montréal, Montréal, Quebec, Canada.

IL's Present address is: Technion-Israel Institute of Technology, The Ruth \& Bruce Rappaport Faculty of Medicine, BAT-GALIM, Haifa, Israel.

VL's present address is: Institute for Diabetes and Cancer, IDC Helmholtz Center Munich, Munich, Germany.

1. Marusyk A, Polyak K. Tumor heterogeneity: causes and consequences. Biochim Biophys Acta. 2010;1805(1):105-117.

2. Hanahan D, Weinberg RA. Hallmarks of cancer: the next generation. Cell. 2011;144(5):646-674

3. Koppenol WH, Bounds PL, Dang CV. Otto Warburg's contributions to current concepts of cancer metabolism. Nat Rev Cancer 2011;11(5):325-337.

4. Kroemer G, Pouyssegur J. Tumor cell metabolism: cancer's Achilles' heel. Cancer Cell. 2008;13(6):472-482.

5. Jose C, Bellance N, Rossignol R. Choosing between glycolysis and oxidative phosphorylation: a tumor's dilemma? Biochim Biophys Acta. 2011;1807(6):552-561.

6. Carracedo A, Cantley LC, Pandolfi PP. Cancer metabolism: fatty acid oxidation in the limelight. Nat Rev Cancer. 2013;13(4):227-232.

7. Schafer ZT, et al. Antioxidant and oncogene rescue of metabolic defects caused by loss of matrix attachment. Nature. 2009;461(7260):109-113.

8. Carracedo A, et al. A metabolic prosurvival role for PML in breast cancer. J Clin Invest. 2012;122(9):3088-3100.

9. Samudio I, et al. Pharmacologic inhibition of fatty acid oxidation sensitizes human leukemia cells to apoptosis induction. J Clin Invest. 2010;120(1):142-156.

10. Dirat B, et al. Cancer-associated adipocytes exhibit an activated phenotype and contribute to breast cancer invasion. Cancer Res. 2011;71(7):2455-2465.

11. Dirat B, Bochet L, Escourrou G, Valet P, Muller C. Unraveling the obesity and breast cancer links: a role for cancer-associated adipocytes? Endocr Dev. 2010;19:45-52.

12. Wang YY, et al. Adipose tissue and breast epithelial cells: a dangerous dynamic duo in breast cancer. Cancer Lett. 2012;324(2):142-151.

13. Bochet L, et al. Adipocyte-derived fibroblasts promote tumor progression and contribute to the desmoplastic reaction in breast cancer. Cancer Res. 2013;73(18):5657-5668.

14. Gazi E, Gardner P, Lockyer NP, Hart CA, Brown MD, Clarke NW. Direct evidence of lipid translocation between adipocytes and prostate cancer cells with imaging FTIR microspectroscopy. J Lipid Res. 2007;48(8):1846-1856.

15. Nieman KM, et al. Adipocytes promote ovarian cancer metastasis and provide energy for rapid tumor growth. Nat Med. 2011;17(11):1498-1503.

16. Lafontan M, Langin D. Lipolysis and lipid mobilization in human adipose tissue. Prog Lipid Res. 2009;48(5):275-297.

17. Petruzzelli M, et al. A switch from white to brown fat increases energy expenditure in cancer-associated cachexia. Cell Metab. 2014;20(3):433-447

18. Bochet L, Meulle A, Imbert S, Salles B, Valet P, Muller C. Cancer-associated adipocytes promotes breast tumor radioresistance. Biochem Biophys Res Commun. 2011;411(1):102-106.

19. Hernlund E, et al. Potentiation of chemotherapeutic drugs by energy metabolism inhibitors 2-deoxyglucose and etomoxir. Int $J$ Cancer. 2008;123(2):476-483.

20. Rossignol R, Gilkerson R, Aggeler R, Yamagata K, Remington SJ, Capaldi RA. Energy substrate modulates mitochondrial structure and oxidative capacity in cancer cells. Cancer Res. 2004;64(3):985-993.

21. Pike Winer LS, Wu M. Rapid analysis of glycolytic and oxidative substrate flux of cancer cells in a microplate. PLoS One. 2014;9(10):e109916.

22. Campanella M, et al. Regulation of mitochondrial structure and function by the F1Fo-ATPase inhibitor protein, IF1. Cell Metab. $2008 ; 8(1): 13-25$. 
23. Sánchez-Cenizo L, et al. Up-regulation of the ATPase inhibitory factor 1 (IF1) of the mitochondrial H+-ATP synthase in human tumors mediates the metabolic shift of cancer cells to a Warburg phenotype. J Biol Chem. 2010;285(33):25308-25313

24. Kang HT, Hwang ES. 2-Deoxyglucose: an anticancer and antiviral therapeutic, but not any more a low glucose mimetic. Life Sci. 2006;78(12):1392-1399.

25. Nanni P, de Giovanni C, Lollini PL, Nicoletti G, Prodi G. TS/A: a new metastasizing cell line from a BALB/c spontaneous mammary adenocarcinoma. Clin Exp Metastasis. 1983;1(4):373-380.

26. van Roy F, Berx G. The cell-cell adhesion molecule E-cadherin. Cell Mol Life Sci. 2008;65(23):3756-3788.

27. Peinado H, Olmeda D, Cano A. Snail, Zeb and bHLH factors in tumour progression: an alliance against the epithelial phenotype? Nat Rev Cancer. 2007;7(6):415-428.

28. Zaugg K, et al. Carnitine palmitoyltransferase $1 \mathrm{C}$ promotes cell survival and tumor growth under conditions of metabolic stress Genes Dev. 2011;25(10):1041-1051

29. Linher-Melville K, Zantinge S, Sanli T, Gerstein H, Tsakiridis T, Singh G. Establishing a relationship between prolactin and altered fatty acid $\beta$-oxidation via carnitine palmitoyl transferase 1 in breast cancer cells. BMC Cancer. 2011;11:56.

30. Haemmerle G, et al. Defective lipolysis and altered energy metabolism in mice lacking adipose triglyceride lipase. Science. 2006;312(5774):734-737.

31. Greenberg AS, et al. The role of lipid droplets in metabolic disease in rodents and humans. J Clin Invest. 2011;121(6):2102-2110.

32. Listenberger LL, et al. Triglyceride accumulation protects against fatty acid-induced lipotoxicity. Proc Natl Acad Sci USA. 2003;100(6):3077-3082.

33. Rial E, Rodríguez-Sánchez L, Gallardo-Vara E, Zaragoza P, Moyano E, González-Barroso MM. Lipotoxicity, fatty acid uncoupling and mitochondrial carrier function. Biochim Biophys Acta. 2010;1797(6-7):800-806.

34. Bouillaud F. UCP2, not a physiologically relevant uncoupler but a glucose sparing switch impacting ROS production and glucose sensing. Biochim Biophys Acta. 2009;1787(5):377-383

35. Ayyasamy V, et al. Cellular model of Warburg effect identifies tumor promoting function of UCP2 in breast cancer and its suppression by genipin. PLoS One. 2011;6(9):e24792.

36. Kuai XY, Ji ZY, Zhang HJ. Mitochondrial uncoupling protein 2 expression in colon cancer and its clinical significance. World J Gastroenterol. 2010;16(45):5773-5778.

37. Jiang L, et al. A microRNA-30e/mitochondrial uncoupling protein 2 axis mediates TGF- $\beta 1$-induced tubular epithelial cell extracellular matrix production and kidney fibrosis. Kidney Int. 2013;84(2):285-296.

38. Boukouris AE, Zervopoulos SD, Michelakis ED. Metabolic Enzymes Moonlighting in the Nucleus: Metabolic Regulation of Gene Transcription. Trends Biochem Sci. 2016;41(8):712-730.

39. Zaidi N, Lupien L, Kuemmerle NB, Kinlaw WB, Swinnen JV, Smans K. Lipogenesis and lipolysis: the pathways exploited by the cancer cells to acquire fatty acids. Prog Lipid Res. 2013;52(4):585-589.

40. Cabodevilla AG, et al. Cell survival during complete nutrient deprivation depends on lipid droplet-fueled $\beta$-oxidation of fatty acids. J Biol Chem. 2013;288(39):27777-27788.

41. Banke NH, et al. Preferential oxidation of triacylglyceride-derived fatty acids in heart is augmented by the nuclear receptor PPARalpha. Circ Res. 2010;107(2):233-241.

42. Nomura DK, Long JZ, Niessen S, Hoover HS, Ng SW, Cravatt BF. Monoacylglycerol lipase regulates a fatty acid network that promotes cancer pathogenesis. Cell. 2010;140(1):49-61.

43. Prentki M, Madiraju SR. Glycerolipid metabolism and signaling in health and disease. Endocr Rev. 2008;29(6):647-676.

44. Menendez JA. Fine-tuning the lipogenic/lipolytic balance to optimize the metabolic requirements of cancer cell growth: molecular mechanisms and therapeutic perspectives. Biochim Biophys Acta. 2010;1801(3):381-391.

45. Meulle A, Salles B, Daviaud D, Valet P, Muller C. Positive regulation of DNA double strand break repair activity during differentiation of long life span cells: the example of adipogenesis. PLoS One. 2008;3(10):e3345.

46. Rizki A, et al. A human breast cell model of preinvasive to invasive transition. Cancer Res. 2008;68(5):1378-1387.

47. Weaver VM, et al. Reversion of the malignant phenotype of human breast cells in three-dimensional culture and in vivo by integrin blocking antibodies. J Cell Biol. 1997;137(1):231-245.

48. Vert JP, Foveau N, Lajaunie C, Vandenbrouck Y. An accurate and interpretable model for siRNA efficacy prediction. BMC Bioinformatics. 2006; 7:520.

49. Despras E, et al. Long-term XPC silencing reduces DNA double-strand break repair. Cancer Res. 2007;67(6):2526-2534.

50. Daviaud D, et al. TNFalpha up-regulates apelin expression in human and mouse adipose tissue. FASEB J. 2006;20(9):1528-1530.

51. Attané C, et al. Apelin treatment increases complete Fatty Acid oxidation, mitochondrial oxidative capacity, and biogenesis in muscle of insulin-resistant mice. Diabetes. 2012;61(2):310-320.

52. Muller C, Monferran S, Gamp AC, Calsou P, Salles B. Inhibition of Ku heterodimer DNA end binding activity during granulocytic differentiation of human promyelocytic cell lines. Oncogene. 2001;20(32):4373-4382.

53. Laurent V, et al. Periprostatic adipocytes act as a driving force for prostate cancer progression in obesity. Nat Commun 2016;7:10230 